\title{
News-generated dependence and optimal portfolios for $n$ stocks in a market of Barndorff-Nielsen and Shephard type.
}

\author{
Carl Lindberg \\ Department of Mathematical Statistics \\ Chalmers University of Technology and Göteborg University \\ Göteborg, Sweden.
}

September 28, 2004

\begin{abstract}
We consider Merton's portfolio optimization problem in a Black and Scholes market with non-Gaussian stochastic volatility of Ornstein-Uhlenbeck type. The investor can trade in $n$ stocks and a risk-free bond. We assume that the dependence between stocks lies in that they partly share the Ornstein-Uhlenbeck processes of the volatility. We refer to these as news processes, and interpret this as that dependence between stocks lies solely in their reactions to the same news. We show that this dependence generates covariance, and give statistical methods for both the fitting and verification of the model to data. Using dynamic programming, we derive and verify explicit trading strategies and Feynman-Kac representations of the value function for power utility.
\end{abstract}

\section{Introduction}

A classical problem in mathematical finance is the question of how to optimally allocate capital between different assets. In a Black and Scholes market with constant coefficients, this was solved by Merton in [15] and [16]. Recently, [6] solved the same problem for one stock and a bond in the more general market model of [3]. In [3], Barndorff-Nielsen and Shephard propose modeling the volatility in asset price dynamics as a weighted sum of non-Gaussian OrnsteinUhlenbeck (OU) processes of the form

$$
d y(t)=-\lambda y(t) d t+d z(t)
$$

The author would like to thank his supervisors Holger Rootzén and Fred Espen Benth for valuable discussions, as well as for carefully reading through preliminary versions of this paper. 
where $z$ is a subordinator and $\lambda>0$. This framework is a powerful modeling tool that allows us to capture several of the observed features in financial time series, such as semi-heavy tails, volatility clustering, and skewness. We extend the model by introducing a new dependence structure, in which the dependence between assets lies in that they share some of the OU processes of the volatility. We will refer to the OU processes as news processes, which implies the interpretation that the dependence between financial assets is reactions to the same news. We show that this dependence generates covariance, and give statistical methods for both the fitting and verification of the model to data.

In this extended model we consider an investor who wants to maximize her utility from terminal wealth by investing in $n$ stocks and a bond. This problem is an $n$-stock extension of [6]. We allow for the investor to have restrictions on the fractions of wealth held in each stock, as well as borrowing and short-selling restrictions on the entire portfolio. For simplicity of notation, we have formulated and solved the problem for two stocks and a bond. However, the general case is completely analogous. The stochastic optimization problem is solved via dynamic programming and the associated Hamilton-Jakobi-Bellman (HJB) integro-differential equation. By use of a verification theorem, we identify the optimal expected utility from terminal wealth as the solution of a second-order integro-differential equation. For power utility, we then compute the solution to this equation via a Feynman-Kac representation, and obtain explicit optimal allocation strategies. All results are derived under exponential integrability assumptions on the Lévy measures of the subordinators.

Recently, portfolio optimization under stochastic volatility has been treated in a number of articles. In [9] and [11], Merton's problem is studied with stochastic volatility being modelled as a mean-reverting process. The paper [18] use partial observation to solve a portfolio problem with a stochastic volatility process driven by a Brownian motion correlated to the dynamics of the risky asset. Going beyond the classical geometric Brownian motion, [4], [5], and [8] treat different portfolio problems when the risky assets are driven by Lévy processes, and [12] derive explicit solutions for log-optimal portfolios in terms of the semimartingale characteristics of the price process. For an introduction to the market model of Barndorff-Nielsen and Shephard we refer to [2] and [3]. For option pricing in this context, see [17].

This paper has seven sections. In Section 2 we give a rigorous formulation of the market and the portfolio optimization problem. We also discuss the market model and the implications of the dependence structure. In Section 3 we derive some useful results on the stochastic volatility model, and on moments of the wealth process. We prove our verification theorem in Section 4, and use it in Section 5 to verify the solution we have obtained. Section 6 states our results, without proofs, in the general setting. We discuss our results and future research in Section 7. 


\section{The optimization problem}

In this section we define, and discuss, the market model. We also set up our optimization problem.

\subsection{The market model}

For $0 \leq t \leq T<\infty$, we assume as given a complete probability space $(\Omega, \mathcal{F}, P)$ with a filtration $\left\{\mathcal{F}_{s}\right\}_{t \leq s \leq T}$ satisfying the usual conditions. Introduce $m$ independent subordinators $Z_{j}$, and denote their Lévy measures by $l_{j}(d z), j=$ $1, \ldots, m$. Remember that a subordinator is defined to be a Lévy process taking values in $[0, \infty)$, which implies that its sample paths are increasing. The Lévy measure $l$ of a subordinator satisfies the condition

$$
\int_{0+}^{\infty} \min (1, z) l(d z)<\infty .
$$

We assume that we use the cádlág version of $Z_{j}$. Let $B_{i}, i=1,2$, be two Wiener processes independent of all the subordinators. We now introduce our stochastic volatility model. It is an extension of the model proposed by Barndorff-Nielsen and Shephard in [3] to the case of two stocks, under a special dependence structure. To begin with, our model is identical to theirs. We will discuss the differences as they occur.

The next extension of the model, to $n$ stocks, is only a matter of notation. Denote by $Y_{j}, j=1, \ldots, m$, the OU stochastic processes whose dynamics are governed by

$$
d Y_{j}(t)=-\lambda_{j} Y_{j}(t) d t+d Z_{j}\left(\lambda_{j} t\right)
$$

where the rate of decay is denoted by $\lambda_{j}>0$. The unusual timing of $Z_{j}$ is chosen so that the marginal distribution of $Y_{j}$ will be unchanged regardless of the value of $\lambda_{j}$. To make the OU processes and the Wiener processes simultaneously adapted, we use the filtration

$$
\left\{\sigma\left(B_{1}(s), B_{2}(s), Z_{1}\left(\lambda_{1} s\right), \ldots, Z_{m}\left(\lambda_{m} s\right)\right)\right\}_{t \leq s \leq T} .
$$

From now on we view the processes $Y_{j}, j=1, \ldots, m$ in our model as news processes associated to certain events, and the jump times of $Z_{j}, j=1, \ldots, m$ as news or the release of information on the market. The stationary process $Y_{j}$ is representable as

$$
Y_{j}(s)=\int_{-\infty}^{t} \exp (u) d Z_{j}\left(\lambda_{j} s+u\right), \quad s \geq t,
$$

but can also be written as

$$
Y_{j}(s)=y_{j} e^{-\lambda_{j}(s-t)}+\int_{t}^{s} e^{-\lambda_{j}(s-u)} d Z_{j}\left(\lambda_{j} u\right), \quad s \geq t,
$$

where $y_{j}:=Y_{j}(t)$, and $y_{j}$ has the stationary marginal distribution of the process and is independent of $Z_{j}(s)-Z_{j}(t), s \geq t$. In particular, if $y_{j}=Y_{j}(t) \geq 0$, 
then $Y_{j}(s) \geq 0$, since $Z_{j}$ is non-decreasing. We set $Z_{j}(0)=0, j=1, \ldots, m$, and set $y:=\left(y_{1}, \ldots, y_{m}\right)$. We assume the usual risk-free bond dynamics

$$
d R(t)=r R(t) d t
$$

with interest rate $r>0$. Define the two stocks $S_{1}, S_{2}$ to have the dynamics

$$
d S_{i}(t)=\left(\mu_{i}+\beta_{i} \sigma_{i}(t)\right) S_{i}(t) d t+\sqrt{\sigma_{i}(t)} S_{i}(t) d B_{i}(t) .
$$

Here $\mu_{i}$ are the constant mean rates of return, and $\beta_{i}$ are skewness parameters. We will call $\mu_{i}+\beta_{i} \sigma_{i}(t)$ the mean rate of return for stock $i$ at time $t$. For notational simplicity in our portfolio problem we denote the volatility processes by $\sigma_{i}$ instead of the more customary $\sigma_{i}^{2}$. We define $\sigma_{i}$ as

$$
\sigma_{i}(s):=\sigma_{i}^{t, y}(s):=\sum_{j=1}^{m} \omega_{i, j} Y_{j}(s), \quad s \in[t, T],
$$

where $\omega_{i, j} \geq 0$ are weights summing to one for each $i$. The notation $\sigma_{i}^{t, y}$ denotes conditioning on $Y(t)$. Our model is here not the same as just two separate models of Barndorff-Nielsen and Shephard type. The difference is that the volatility processes depend on the same news processes. These volatility dynamics gives us the stock price processes

$$
S_{i}(s)=S_{i}(t) \exp \left(\int_{t}^{s}\left(\mu_{i}+\left(\beta_{i}-\frac{1}{2}\right) \sigma_{i}(u)\right) d u+\int_{t}^{s} \sqrt{\sigma_{i}(u)} d B_{i}(u)\right) .
$$

This stock price model does not have statistically independent increments and it is non-stationary. It also allows for the increments of the returns $R_{i}(t):=$ $\log \left(S_{i}(t) / S_{i}(0)\right), i=1,2$, to have semi-heavy tails as well as both volatility clustering and skewness. The increments of the returns $R_{i}$ are stationary since

$$
R_{i}(s)-R_{i}(t)=\log \left(\frac{S_{i}(s)}{S_{i}(0)}\right)-\log \left(\frac{S_{i}(t)}{S_{i}(0)}\right)=\log \left(\frac{S_{i}(s)}{S_{i}(t)}\right)={ }^{\mathcal{L}} R_{i}(s-t),
$$

where $"={ }^{\mathcal{L}} "$ denotes equality in law.

\subsection{Discussion of the market model}

This section aims to show that the dependence structure proposed in Section 2.1 is not only simple from a statistical point of view, but also has very appealing economical interpretations.

The paper [3] suggests a model with $n$ stocks with dynamics

$$
d S(t)=\{\mu+\beta \Sigma(t)\} S(t) d t+\Sigma(t)^{\frac{1}{2}} S(t) d B(t),
$$

where $\Sigma$ is a time-varying stochastic volatility matrix, $\mu$ and $\beta$ are vectors, and $B$ is a vector of independent Wiener processes. This model includes ours as a special case with $\Sigma$ being a diagonal matrix. However, in the classical Black 
and Scholes market, dependence is modelled by covariance. In the case of two stocks this means that for $s \geq t$,

$$
S_{1}(s)=S_{1}(t) \exp \left(\left(\mu_{1}-\frac{1}{2} \sigma_{11}-\frac{1}{2} \sigma_{12}\right)(s-t)+\sqrt{\sigma_{11}} B_{1}(s)+\sqrt{\sigma_{12}} B_{2}(s)\right)
$$

and

$$
S_{2}(s)=S_{2}(t) \exp \left(\left(\mu_{2}-\frac{1}{2} \sigma_{21}-\frac{1}{2} \sigma_{22}\right)(s-t)+\sqrt{\sigma_{21}} B_{1}(s)+\sqrt{\sigma_{22}} B_{2}(s)\right)
$$

for a volatility matrix $\sigma$, and $B_{1}(t)=B_{2}(t)=0$.

In our model, stock prices develop independently beside from reacting to the same news. From an economic viewpoint, one can expect the model parameters to be more stable than in the classical Black and Scholes market. For example, we do not require stability over expected rate of return. Instead we ask that every time the market is "nervous" to a certain degree, i.e. for every specific value of the volatility $\sigma_{i}$, the mean rate of return $\mu_{i}+\beta_{i} \sigma_{i}$ will be the same. We can interpret this as that we only need stability in how the market reacts to news.

As we will see, for the purpose of portfolio optimization we do not need to know the weights $\omega_{i, j}$. More importantly, the model generates a non-diagonal covariance matrix for the increments of the returns over the same time period, which is the most frequently used measure of dependence in finance. Since the returns have stationary increments, it is sufficient to show this result for $R_{i}$, $i=1,2$. Note that we have

$$
\begin{aligned}
& \operatorname{Cov}\left(R_{1}(s)-R_{1}(t), R_{2}(u)-R_{2}(v)\right) \\
& \begin{array}{r}
\operatorname{Cov}\left(R_{1}(s), R_{2}(u)\right)-\operatorname{Cov}\left(R_{1}(s), R_{2}(v)\right) \\
\quad-\operatorname{Cov}\left(R_{1}(t), R_{2}(u)\right)+\operatorname{Cov}\left(R_{1}(t), R_{2}(v)\right),
\end{array}
\end{aligned}
$$

for $s, t, u, v \in[0, T]$. As will be shown below, for $s, t \in[0, T]$, we have that

$$
\begin{aligned}
\operatorname{Cov}\left(R_{1}(s), R_{2}(t)\right) \\
=\left(\beta_{1}-\frac{1}{2}\right)\left(\beta_{2}-\frac{1}{2}\right) \sum_{j=1}^{m} \omega_{1, j} \omega_{2, j} \operatorname{Var}\left(Y_{j}(0)\right) \\
\quad \times \frac{e^{-\lambda_{j} s}+e^{-\lambda_{j} t}-e^{-\lambda_{j}|s-t|}-1+2 \lambda_{j} \min (s, t)}{\lambda_{j}^{2}},
\end{aligned}
$$

which for $s=t$ simplifies to

$$
\begin{aligned}
& \operatorname{Cov}\left(R_{1}(t), R_{2}(t)\right) \\
& =2\left(\beta_{1}-\frac{1}{2}\right)\left(\beta_{2}-\frac{1}{2}\right) \sum_{j=1}^{m} \omega_{1, j} \omega_{2, j} \operatorname{Var}\left(Y_{j}(0)\right) \frac{e^{-\lambda_{j} t}-1+\lambda_{j} t}{\lambda_{j}^{2}} .
\end{aligned}
$$

This result says that the model generates a covariance matrix between returns, but we do not immediately know which correlations that can be obtained. It 
turns out that we can get correlations $\operatorname{Corr}\left(R_{1}(t), R_{2}(t)\right)$ in the entire interval $(-1,1)$.

To derive Equation (2.6), by definition of $\sigma_{i}$ we have that

$$
\begin{aligned}
& \mathbb{E}\left[R_{1}(s) R_{2}(t)\right] \\
& =\mathbb{E}\left[\left(\int_{0}^{s} \mu_{1}+\left(\beta_{1}-\frac{1}{2}\right) \sigma_{1}(u) d u+\int_{0}^{s} \sqrt{\sigma_{1}(u)} d B_{1}(u)\right)\right. \\
& \left.\quad \times\left(\int_{0}^{t} \mu_{2}+\left(\beta_{2}-\frac{1}{2}\right) \sigma_{2}(u) d u+\int_{0}^{t} \sqrt{\sigma_{2}(u)} d B_{2}(u)\right)\right] \\
& =\mu_{1} \mu_{2} s t+\mu_{1} s\left(\beta_{2}-\frac{1}{2}\right) \sum_{j=1}^{m} \omega_{2, j} \mathbb{E}\left[\int_{0}^{t} Y_{j}(u) d u\right] \\
& \quad+\mu_{2} t\left(\beta_{1}-\frac{1}{2}\right) \sum_{j=1}^{m} \omega_{1, j} \mathbb{E}\left[\int_{0}^{s} Y_{j}(u) d u\right] \\
& \quad+\left(\beta_{1}-\frac{1}{2}\right)\left(\beta_{2}-\frac{1}{2}\right) \sum_{i, j=1}^{m} \omega_{1, i} \omega_{2, j} \mathbb{E}\left[\int_{0}^{s} Y_{i}(u) d u \int_{0}^{t} Y_{j}(u) d u\right] .
\end{aligned}
$$

Similarly,

$$
\mathbb{E}\left[R_{1}(t)\right]=\mu_{1} t+\left(\beta_{1}-\frac{1}{2}\right) \sum_{j=1}^{m} \omega_{1, j} \mathbb{E}\left[\int_{0}^{t} Y_{j}(u) d u\right] .
$$

This gives that

$$
\begin{aligned}
& \operatorname{Cov}\left(R_{1}(s), R_{2}(t)\right) \\
& =\left(\beta_{1}-\frac{1}{2}\right)\left(\beta_{2}-\frac{1}{2}\right) \sum_{j=1}^{m} \omega_{1, j} \omega_{2, j} \operatorname{Cov}\left(\int_{0}^{s} Y_{j}(u) d u, \int_{0}^{t} Y_{j}(u) d u\right) .
\end{aligned}
$$

By stationarity, we have that $\mathbb{E}\left[Y_{j}(t)\right]=\mu_{Y_{j}}$, for some constant $\mu_{Y_{j}}>0$, for all $t \in \mathbb{R}$. If we assume that $u \leq v$, the independence of the increments of $Y_{j}$ gives that

$$
\begin{aligned}
& \operatorname{Cov}\left(Y_{j}(u), Y_{j}(v)\right) \\
& =\mathbb{E}\left[\left(Y_{j}(u)-\mu_{Y_{j}}\right)\left(Y_{j}(v)-\mu_{Y_{j}}\right)\right] \\
& =\mathbb{E}\left[e^{-\lambda_{j}(v-u)} Y_{j}(u)^{2}+Y_{j}(u) \int_{u}^{v} e^{-\lambda_{j}(v-s)} d Z\left(\lambda_{j} s\right)\right]-\mu_{Y_{j}}^{2} \\
& =e^{-\lambda_{j}(v-u)} \mathbb{E}\left[Y_{j}(0)^{2}\right]-e^{-\lambda_{j}(v-u)} \mu_{Y_{j}}^{2} \\
& =e^{-\lambda_{j}(v-u)} \operatorname{Var}\left(Y_{j}(0)\right) .
\end{aligned}
$$

The same calculations for $v \leq u$ shows that

$$
\operatorname{Cov}\left(Y_{j}(u), Y_{j}(v)\right)=e^{-\lambda_{j}|v-u|} \operatorname{Var}\left(Y_{j}(0)\right),
$$


and we get

$$
\begin{aligned}
& \operatorname{Cov}\left(\int_{0}^{s} Y_{j}(u) d u, \int_{0}^{t} Y_{j}(u) d u\right) \\
& =\int_{0}^{s} \int_{0}^{t} \operatorname{Cov}\left(Y_{j}(u), Y_{j}(v)\right) d u d v \\
& =\operatorname{Var}\left(Y_{j}(0)\right) \frac{e^{-\lambda_{j} s}+e^{-\lambda_{j} t}-e^{-\lambda_{j}|s-t|}-1+2 \lambda_{j} \min (s, t)}{\lambda_{j}^{2}} .
\end{aligned}
$$

By Itô's isometry (see [22]) we get, similar to above,

$$
\operatorname{Var}\left(R_{i}(t)\right)=\sum_{j=1}^{m}\left(2\left(\beta_{i}-\frac{1}{2}\right)^{2} \omega_{i, j}^{2} \operatorname{Var}\left(Y_{j}(0)\right) \frac{e^{-\lambda_{j} t}-1+\lambda_{j} t}{\lambda_{j}^{2}}+\omega_{i, j} \mu_{Y_{j}} t\right)
$$

for $i=1,2$. This gives

$$
\begin{aligned}
& \operatorname{Corr}\left(R_{1}(s), R_{2}(t)\right) \\
& =\frac{1}{2} \frac{\left(\beta_{1}-\frac{1}{2}\right)\left(\beta_{2}-\frac{1}{2}\right)}{\left|\beta_{1}-\frac{1}{2}\right|\left|\beta_{2}-\frac{1}{2}\right|} \sum_{j=1}^{m} \omega_{1, j} \omega_{2, j} \operatorname{Var}\left(Y_{j}(0)\right) \\
& \times \frac{e^{-\lambda_{j} s}+e^{-\lambda_{j} t}-e^{-\lambda_{j}|s-t|}-1+2 \lambda_{j} \min (s, t)}{\lambda_{j}^{2}} \\
& \times \frac{1}{\sqrt{\sum_{j=1}^{m}\left(\omega_{1, j}^{2} \operatorname{Var}\left(Y_{j}(0)\right) \frac{e^{-\lambda_{j} s}-1+\lambda_{j} s}{\lambda_{j}^{2}}+\frac{\omega_{1, j} \mu_{Y_{j}} s}{2\left(\beta_{1}-\frac{1}{2}\right)^{2}}\right)}} \\
& \times \frac{1}{\sqrt{\sum_{j=1}^{m}\left(\omega_{2, j}^{2} \operatorname{Var}\left(Y_{j}(0)\right) \frac{e^{-\lambda_{j} t}-1+\lambda_{j} t}{\lambda_{j}^{2}}+\frac{\omega_{2, j} \mu_{Y_{j}} t}{2\left(\beta_{2}-\frac{1}{2}\right)^{2}}\right)}},
\end{aligned}
$$

and, for $s=t$,

$$
\begin{aligned}
& \operatorname{Corr}\left(R_{1}(t), R_{2}(t)\right) \\
& =\frac{\left(\beta_{1}-\frac{1}{2}\right)\left(\beta_{2}-\frac{1}{2}\right)}{\left|\beta_{1}-\frac{1}{2}\right|\left|\beta_{2}-\frac{1}{2}\right|} \sum_{j=1}^{m} \omega_{1, j} \omega_{2, j} \operatorname{Var}\left(Y_{j}(0)\right) \frac{e^{-\lambda_{j} t}-1+\lambda_{j} t}{\lambda_{j}^{2}} \\
& \times \frac{1}{\sqrt{\sum_{j=1}^{m}\left(\omega_{1, j}^{2} \operatorname{Var}\left(Y_{j}(0)\right) \frac{e^{-\lambda_{j} t}-1+\lambda_{j} t}{\lambda_{j}^{2}}+\frac{\omega_{1, j} \mu_{Y_{j}} t}{2\left(\beta_{1}-\frac{1}{2}\right)^{2}}\right)}} \\
& \times \frac{1}{\sqrt{\sum_{j=1}^{m}\left(\omega_{2, j}^{2} \operatorname{Var}\left(Y_{j}(0)\right) \frac{e^{-\lambda_{j} t}-1+\lambda_{j} t}{\lambda_{j}^{2}}+\frac{\omega_{2, j} \mu_{Y_{j}} t}{2\left(\beta_{2}-\frac{1}{2}\right)^{2}}\right)}} .
\end{aligned}
$$


There is always a trade-off between accuracy and applicability when designing models. An obvious advantage of our model is that we do not have to estimate a stochastic volatility matrix, and hence we need less data to obtain good estimates of the model parameters. A drawback is that, to obtain high correlations, we need the model to be very skew. This might not fit observed data. It remains to see if the trade-off was a good one.

\subsection{Statistical methodology}

In this section we describe a methodology for fitting the model to return data. We will do this for a Normalized Inverse Gaussian distribution $(N I G)$, which has been shown to fit financial data well, see e.g. [1], [7] and [20]. Our choice plays no formal role in the analysis. We assume that we are observing $R_{i}(\Delta), R_{i}(2 \Delta)-R_{i}(\Delta), \ldots, R_{i}(k \Delta)-R_{i}((k-1) \Delta)$, where $\Delta$ is one day, and $k+1$ is the number of consecutive trading days in our period of observation.

The $N I G$-distribution has parameters $\alpha=\sqrt{\beta^{2}+\gamma^{2}}, \beta, \mu$, and $\delta$. Its density function is

$$
\begin{aligned}
& f_{N I G}(x ; \alpha, \beta, \mu, \delta) \\
& =\frac{\alpha}{\pi} \exp \left(\delta \sqrt{\alpha^{2}-\beta^{2}}-\beta \mu\right) q\left(\frac{x-\mu}{\delta}\right)^{-1} K_{1}\left(\delta \alpha q\left(\frac{x-\mu}{\delta}\right)\right) e^{\beta x}
\end{aligned}
$$

where $q(x)=\sqrt{1+x^{2}}$ and $K_{1}$ denotes the modified Bessel function of the third kind with index 1 . The domain of the parameters is $\mu \in \mathbb{R}, \delta>0$, and $0 \leq|\beta| \leq \alpha$.

A standard result is that if we take $\sigma$ to have an Inverse Gaussian distribution $(I G)$, and draw a $N(0,1)$-distributed random variable $\varepsilon$, then $x=\mu+\beta \sigma+\sqrt{\sigma} \varepsilon$ will be $N I G$-distributed. The $I G$-distribution has density function

$$
f_{I G}(x ; \delta, \gamma)=\frac{\delta}{\sqrt{2 \pi}} \exp (\delta \gamma) x^{-\frac{3}{2}} \exp \left(-\frac{1}{2}\left(\delta^{2} x^{-1}+\gamma^{2} x\right)\right), \quad x>0,
$$

where $\delta$ and $\gamma$ are the same as in the $N I G$-distribution. The existence and integrability of Lévy measures $l_{j}$ such that the volatility processes $\sigma_{i}$ will have $I G$-distributed marginals is not obvious. See [2] and [21, Section 17] for this theory. The Lévy density $l$ of the subordinator $Z$ of an $I G$-distributed news process $Y$ is

$$
l(x)=(2 \pi)^{-\frac{1}{2}} \frac{\delta}{2}\left(x^{-1}+\gamma^{2}\right) x^{-\frac{1}{2}} e^{-\frac{\gamma^{2} x}{2}},
$$

where $(\delta, \gamma)$ are the parameters of the $I G$-distribution.

The method described in [3], which we will further extend, uses that the marginal distribution of the volatility processes $\sigma_{i}$ are invariant to the rates of decay $\lambda_{j}$. These parameters $\lambda_{j}$ are then used to fit the autocorrelation function of the $\sigma_{i}$ to log-return data. The autocorrelation $\rho$ is defined by

$$
\rho_{\sigma}(h)=\frac{\operatorname{Cov}(\sigma(h), \sigma(0))}{\operatorname{Var}(\sigma(0))}, \quad h \in \mathbb{R} .
$$


For simplicity of exposition we will assume that we only need one $\lambda$ to correctly model the autocorrelation function of both stocks. However, for reasons to be explained later, we will assume that $m=3$, and that all $\lambda_{1}=\lambda_{2}=\lambda_{3}=\lambda$. For our model calculations show that, for general $m$,

$$
\rho_{\sigma_{i}}(h)=\omega_{i, 1} \exp \left(-\lambda_{1}|h|\right)+\ldots+\omega_{i, m} \exp \left(-\lambda_{m}|h|\right),
$$

where the $\omega_{i, j} \geq 0$, are the weights from the volatility processes that sum to one. Observe that since we have assumed the rates of decay $\lambda_{j}$ to be equal, we immediately get that $\rho_{\sigma_{i}}(h)=\exp (-\lambda|h|)$. We proved this more simple result in Subsection 2.2. The proof of the general case is analogous.

We assume that we have fitted $N I G$-distributions to the empirical marginal distributions of two stocks, and that we have found a $\lambda$ such that our model has the right autocorrelation function. This can be done by empirically calculating the autocorrelation functions $\rho_{\sigma_{i}}(h)$ for different values of $h$, and then find a $\lambda$ so that the theoretical and empirical autocorrelation functions match. We denote the $I G$-parameters of the volatility processes $\sigma_{i}$ by $\left(\delta_{i}, \gamma_{i}\right), i=1,2$. By Equation (2.7) we can now fit the covariance of the model to the empirical covariance from the return data. This can be done by letting the two stocks "share" the news process $Y_{3}$, and each have one of the news processes $Y_{i}, i=1,2$, "of their own." In general, this is done for each rate of decay. We formulate this mathematically as

$$
\begin{gathered}
\sigma_{1}=\omega_{1,1} Y_{1}+\omega_{1,3} Y_{3} \sim I G\left(\delta_{1}, \gamma_{1}\right) \\
\sigma_{2}=\omega_{2,1} Y_{2}+\omega_{2,3} Y_{3} \sim I G\left(\delta_{2}, \gamma_{2}\right) .
\end{gathered}
$$

We now state two properties of $I G$-distributed random variables that we will need below. For $X \sim I G\left(\delta_{X}, \gamma_{X}\right)$, we have that

$$
a X \sim I G\left(a^{\frac{1}{2}} \delta_{X}, a^{-\frac{1}{2}} \gamma_{X}\right) .
$$

Furthermore, if $Y \sim I G\left(\delta_{Y}, \gamma_{Y}\right)$ and is independent of $X$ and we assume that $\gamma_{X}=\gamma_{Y}=: \gamma$, we have that $X+Y \sim I G\left(\delta_{X}+\delta_{Y}, \gamma\right)$. Because of this formula we can let

$$
\begin{aligned}
\omega_{1,1} Y_{1} & \sim I G\left(\delta_{1,1}, \gamma_{1}\right) \\
\omega_{1,3} Y_{3} & \sim I G\left(\delta_{1,3}, \gamma_{1}\right),
\end{aligned}
$$

where

$$
\delta_{1,1}+\delta_{1,3}=\delta_{1},
$$

and

$$
\begin{aligned}
\omega_{2,1} Y_{2} & \sim I G\left(\delta_{2,1}, \gamma_{2}\right) \\
\omega_{2,3} Y_{3} & \sim I G\left(\delta_{2,3}, \gamma_{2}\right),
\end{aligned}
$$

where

$$
\delta_{2,1}+\delta_{2,3}=\delta_{2},
$$


We see, by the scaling property of the $I G$-distribution, that the two expressions for the distribution of $Y_{3}$,

$$
Y_{3} \sim I G\left(\omega_{1,3}^{-\frac{1}{2}} \delta_{1,3}, \omega_{1,3}^{\frac{1}{2}} \gamma_{1}\right),
$$

and

$$
Y_{3} \sim I G\left(\omega_{2,3}^{-\frac{1}{2}} \delta_{2,3}, \omega_{2,3}^{\frac{1}{2}} \gamma_{2}\right),
$$

must be identical. With the aid of Equation (2.8), in which we use that the variance $\operatorname{Var}(Y(0))$ of a stationary inverse Gaussian process $Y$ is $\delta / \gamma^{3}$, we see that Equation (2.7) becomes

$$
2\left(\beta_{1}-\frac{1}{2}\right)\left(\beta_{2}-\frac{1}{2}\right) \frac{\omega_{1,3}}{\omega_{2,3}} \frac{\delta_{2,3}}{\gamma_{2}^{3}} \frac{e^{-\lambda_{j} \Delta}-1+\lambda_{j} \Delta}{\lambda_{j}^{2}}=C
$$

where $C$ is the covariance that we want the returns to have. It is now straightforward to check that there are non-unique choices of $\omega_{i, j}$ such that we can obtain both the right autocorrelation function of $\sigma_{i}$ and a specific covariance for the returns. The autocorrelation function parameter $\lambda$ is already correct by assumption, and we constructed the news processes $Y_{j}$ so that their marginal distribution would not depend on it. Hence we only have to take care of the covariance of the returns $R_{i}$. We do this by using Equations $(2.9), \ldots,(2.13)$. Note that there is nothing crucial in our choice of covariance as measure of dependence, nor does it matter how many different rates of decay we use.

We now give a simple approach to determine how well our model captures the true covariance. We begin by fitting a marginal distribution to return data, thereby obtaining the parameters $\mu_{i}$ and $\beta_{i}, i=1,2$. Since we have that the return processes $R_{i}, i=1,2$, are semimartingales, their quadratic variations, denoted by [.] , are $\int_{t}^{s} \sigma_{i}(u) d u, s \geq t$. That is, for a sequence of random partitions tending to the identity, we have

$$
\left[\log \left(S_{i} / S_{i}(t)\right)\right](s)=\int_{t}^{s} \sigma_{i}(u) d u
$$

where convergence is uniformly on compacts in probability. This is a standard result in stochastic calculus. For each trading day we now empirically calculate the integrated volatility, that is, we calculate the quadratic variation of the observed returns over a trading day and, by the formula above, use that as a constant approximation of the volatility during that day. If we do this for a number of trading days, we get approximations of the volatility processes $\sigma_{i}$ for that period of time. Using the fitted parameters $\mu_{i}, \beta_{i}$ and generated $N(0,1)$-distributed variables in Equation $(2.5)$, we can now simulate "alternative" returns. We then calculate the covariance-matrix of both the return data set and the simulated alternative returns and compare them statistically. 


\subsection{The control problem}

A main purpose of this paper is to find trading strategies that optimizes the trader's expected utility from wealth in a deterministic future point in time. The utility is measured by a utility function $U$ chosen by the trader. This utility function $U$ is a measure of the trader's aversion towards risk, in that it concretizes how much the trader is willing to risk to obtain a certain level of wealth. Our approach to finding these trading strategies, and the value function $V$, is dynamic programming and stochastic control. We will make use of many of the results found in [6], since most of their ideas are applicable in our setting. However, we need to adapt their results to our case.

In this section we set up the control problem under the stock price dynamics of Equation (2.3). Recall that $\sigma_{1}$ and $\sigma_{2}$, are weighted sums of the news processes, see Equation (2.4). We begin by defining a value function $V$ as the maximum amount of expected utility that we can obtain from a trading strategy, given a certain amount of capital. We then set up the associated Hamilton-Jakobi-Bellman equation of the value function $V$. This equation is a central part of our problem, as it is, in a sense, an optimality condition. Most of the later sections will be devoted to finding and verifying solutions to it.

Denote by $\pi_{i}(t)$ the fraction of wealth invested in stock $i$ at time $t$, and set $\pi=\left(\pi_{1}, \pi_{2}\right)$. The fraction of wealth held in the risk-free asset is $\left(1-\pi_{1}-\pi_{2}\right)$. We allow no short-selling of stocks or bond, which implies the conditions $\pi_{i} \in$ $[0,1], i=1,2$, and $\pi_{1}+\pi_{2} \leq 1$, a.s., for all $t \leq s \leq T$. However, these restrictions are partly for mathematical convenience. We could equally well have chosen constants $a_{i}, b_{i}, c, d \in \mathbb{R}, a_{i}<b_{i}, c<d$, such that the constraints would have taken the form $\pi_{i} \in\left[a_{i}, b_{i}\right], i=1,2$, and $c \leq \pi_{1}+\pi_{2} \leq d$, a.s., for all $t \leq s \leq T$. The analysis is analogous in this case, but more notationally complex. This general setting allows us to consider, for example, law enforced restrictions on the fraction of wealth held in a specific stock, as well as short-selling and borrowing of capital. We state the main results in this setting, further generalized to $n$ stocks, in Section 6 .

The wealth process $W$ is defined as

$$
\begin{array}{r}
W(s)=\frac{\pi_{1}(s) W(s)}{S_{1}(s)} S_{1}(s)+\frac{\pi_{2}(s) W(s)}{S_{2}(s)} S_{2}(s) \\
+\frac{\left(1-\pi_{1}(s)-\pi_{2}(s)\right) W(s)}{R(s)} R(s),
\end{array}
$$

where $\pi_{i}(s) W(s) / S_{i}(s)$ is the number of shares of stock $i$ which is held at time $s$. We also assume that the portfolio needs to be self-financing in the sense that no capital is entered or withdrawn. This can be formulated mathematically as

$W(s)=W(t)+\sum_{i=1}^{2} \int_{t}^{s} \frac{\pi_{i}(u) W(u)}{S_{i}(u)} d S_{i}(u)+\int_{t}^{s} \frac{\left(1-\pi_{1}(u)-\pi_{2}(u)\right) W(u)}{R(u)} d R(u)$,

for all $s \in[t, T]$. See [14] for a motivating discussion. The self-financing condi- 
tion gives the wealth dynamics for $t \leq s \leq T$ as

$$
\begin{aligned}
d W(s)=W & (s) \pi_{1}(s)\left(\mu_{1}+\beta_{1} \sigma_{1}(s)-r\right) d s \\
& +W(s) \pi_{2}(s)\left(\mu_{2}+\beta_{2} \sigma_{2}(s)-r\right) d s+r W(s) d s \\
& +\pi_{1}(s) \sqrt{\sigma_{1}(s)} W(s) d B_{1}(s)+\pi_{2}(s) \sqrt{\sigma_{2}(s)} W(s) d B_{2}(s),
\end{aligned}
$$

with initial wealth $W(t)=w$.

Our definition of the set of admissible controls now seems natural.

Definition 2.1 The set $\mathcal{A}_{t}$ of admissible controls is given by $\mathcal{A}_{t}:=\{\pi=$ $\left(\pi_{1}, \pi_{2}\right): \pi_{i}$ is progressively measurable, $\pi_{i}(s) \in[0,1], i=1,2$, and $\pi_{1}+\pi_{2} \leq 1$ a.s. for all $t \leq s \leq T$, and a unique solution $W^{\pi}$ of Equation (2.14) exists $\}$.

An investment strategy $\pi=\{\pi(s): t \leq s \leq T\}$ is said to be admissible if $\pi \in \mathcal{A}_{t}$. Later we will need some exponential integrability conditions on the Lévy measures. We therefore assume that the following holds:

Condition 2.1 For a constant $c_{j}>0$ to be specified below,

$$
\int_{0+}^{\infty}\left(e^{c_{j} z}-1\right) l_{j}(d z)<\infty, j=1, \ldots, m .
$$

Recall that the Lévy density $l$ of the subordinator $Z$ of an $I G$-distributed news process $Y$ is

$$
l(x)=(2 \pi)^{-\frac{1}{2}} \frac{\delta}{2}\left(x^{-1}+\gamma^{2}\right) x^{-\frac{1}{2}} e^{-\frac{\gamma^{2} x}{2}},
$$

where $(\delta, \gamma)$ are the parameters of the $I G$-distribution. Hence Condition 2.1 is satisfied for $c_{j} \leq \gamma^{2} / 2$.

We know from the theory of subordinators that we have

$$
\mathbb{E}\left[e^{a Z_{j}\left(\lambda_{j} t\right)}\right]=\exp \left(\lambda_{j} \int_{0+}^{\infty}\left(e^{a z}-1\right) l_{j}(d z) t\right)
$$

as long as $a \leq c_{j}$ with $c_{j}$ from Condition 2.1 holds.

Denote $(0, \infty)$ by $\mathbb{R}_{+}$and $[0, \infty)$ by $\mathbb{R}_{0+}$, and assume that $y=\left(y_{1}, \ldots, y_{m}\right) \in$ $\mathbb{R}_{0+}^{m}$. Define the domain $D$ by

$$
D:=\left\{(w, y) \in \mathbb{R}_{+} \times \mathbb{R}_{0+}^{m}\right\} .
$$

We will seek to maximize the functional

$$
J(t, w, y ; \pi)=\mathbb{E}^{t, w, y}\left[U\left(W^{\pi}(T)\right)\right],
$$

where the notation $\mathbb{E}^{t, w, y}$ means expectation conditioned by $W(t)=w$, and $Y_{j}(t)=y_{j}, j=1, \ldots, m$. The function $U$ is the investor's utility function. It is assumed to be concave, non-decreasing, bounded from below, and of sublinear growth in the sense that there exists positive constants $k$ and $\gamma \in(0,1)$ so that 
$U(w) \leq k\left(1+w^{\gamma}\right)$ for all $w \geq 0$. Hence our stochastic control problem is to determine the value function

$$
V(t, w, y)=\sup _{\pi \in \mathcal{A}_{t}} J(t, w, y ; \pi),(t, w, y) \in[0, T] \times \bar{D},
$$

and an investment strategy $\pi^{*} \in \mathcal{A}_{t}$, the optimal investment strategy, such that

$$
V(t, w, y)=J\left(t, w, y ; \pi^{*}\right) .
$$

The HJB equation associated to our stochastic control problem is

$$
\begin{aligned}
0=v_{t} & +\max _{\substack{\pi_{i} \in[0,1], i=1,2, \pi_{1}+\pi_{2} \leq 1}}\left\{\left(\pi_{1}\left(\mu_{1}+\beta_{1} \sigma_{1}-r\right)+\pi_{2}\left(\mu_{2}+\beta_{2} \sigma_{2}-r\right)\right) w v_{w}\right. \\
& \left.+\frac{1}{2}\left(\pi_{1}^{2} \sigma_{1}+\pi_{2}^{2} \sigma_{2}\right) w^{2} v_{w w}\right\}+r w v_{w}-\sum_{j=1}^{m} \lambda_{j} y_{j} v_{y_{j}} \\
& +\sum_{j=1}^{m} \lambda_{j} \int_{0}^{\infty}\left(v\left(t, w, y+z \cdot e_{j}\right)-v(t, w, y)\right) l_{j}(d z)
\end{aligned}
$$

for $(t, w, y) \in[0, T) \times D$. We observe that we have the terminal condition

$$
V(T, w, y)=U(w), \quad \text { for all }(w, y) \in \bar{D},
$$

and the boundary condition

$$
V(t, 0, y)=U(0), \quad \text { for all }(t, y) \in[0, T] \times \mathbb{R}_{0+}^{m} .
$$

We now give a formal motivation to this equation. The HJB equation is obtained by setting the supremum of the "infinitesimal generator" $\mathcal{A}$ of $(W, Y)$ applied to the value function $V$ to zero. In other words, if we assume that $V \in C^{1,2}$, the HJB equation is

$$
\begin{aligned}
& \max _{\substack{\pi_{i} \in[0,1], i=1,2, \pi_{1}+\pi_{2} \leq 1}}(\mathcal{A} V)(w, y) \\
= & \max _{\substack{\pi_{i} \in[0,1], i=1,2, \pi_{1}+\pi_{2} \leq 1}}\left\{\lim _{t \downarrow 0} \frac{\mathbb{E}[V(t, W(t), Y(t))]-V(0, w, y)}{t}\right\}=0,
\end{aligned}
$$

where we have used the definition of $\mathcal{A}$ in the first equality, and Itô's formula (see [19]) to evaluate $\mathbb{E}[V(t, W(t), Y(t))]$. If we denote the continuous part of the quadratic covariation by $[\cdot, \cdot]^{c}$, use the notation $V(t)$ for $V(t, W(t), Y(t))$, 
and set $\Delta X(t):=X(t)-X(t-)$, Itô's formula gives that

$$
\begin{aligned}
& V(t)-V(0) \\
& =\sum_{j=1}^{m} \int_{0+}^{t} V_{y_{j}}(s-) d Y_{j}(s)+\int_{0+}^{t} V_{w}(s-) d W(s)+\int_{0+}^{t} V_{t}(s-) d s \\
& \quad+\frac{1}{2} \int_{0+}^{t} V_{w w}(s-) d[W, W]^{c}(s) \\
& \quad+\sum_{0 \leq s \leq t}\left\{V(s)-V(s-)-\sum_{j=1}^{m} V_{y_{j}}(s-) \Delta Y_{j}(s)\right\} .
\end{aligned}
$$

Let $N_{j}$ denote the Poisson random measure in the Lévy-Khintchine representation of $Z_{j}$. We have used that $\left[Y_{j}, Y_{j}\right]^{c}=0, j=1, \ldots, m$, by Theorem 26 in [19]. The Kunita-Watanabe inequality (see [19, p. 69]) tells us that $d\left[X, Y_{j}\right]^{c}$ is a.e.(path by path) absolutely continuous with respect to $d\left[Y_{j}, Y_{j}\right]^{c}, j=1, \ldots, m$, for a semimartingale $X$. Equations (2.1) and (2.14) now give Equation (2.17) once we have seen that, under quite general integrability conditions,

$$
\begin{aligned}
& \mathbb{E}\left[\sum_{0 \leq s \leq t}\{V(s, W(s), Y(s))-V(s-, W(s-), Y(s-))\}\right] \\
& =\mathbb{E}\left[\sum_{j=1}^{m} \int_{0}^{t} \int_{0+}^{\infty} V\left(s, W(s), Y(s-)+z \cdot e_{j}\right)-V(s, W(s), Y(s-)) N_{j}\left(\lambda_{j} d s, d z\right)\right] \\
& =\sum_{j=1}^{m} \int_{0}^{t} \lambda_{j} \int_{0+}^{\infty} \mathbb{E}\left[V\left(s, W(s), Y(s)+z \cdot e_{j}\right)-V(s, W(s), Y(s))\right] l_{j}(d z) d s,
\end{aligned}
$$

where we have used Fubini-Tonelli's theorem and the fact that, for Borel sets $\Lambda, N_{j}(t, \Lambda)-t l_{j}(\Lambda)$ is a martingale, $j=1, \ldots, m$.

\section{Preliminary estimates}

This section aims at relating the existence of exponential moments of $Y$ to exponential integrability conditions on the Lévy measures, as well as developing moment estimates for the wealth process and showing that the value function is well-defined.

Lemma 3.1 Assume Condition 2.1 holds with $c_{j}=\xi_{j} / \lambda_{j}$ for $\xi_{j}>0$. Then

$\mathbb{E}\left[\exp \left(\xi_{j} \int_{t}^{s} Y_{j}(u) d u\right)\right] \leq \exp \left(\frac{\xi_{j}}{\lambda j} y_{j}+\lambda_{j} \int_{0+}^{\infty}\left\{\exp \left(\frac{\xi_{j} z}{\lambda j}\right)-1\right\} l_{j}(d z)(s-t)\right)$ 
Proof. We get from the dynamics (2.1) of $Y_{j}$ that

$$
\begin{aligned}
\lambda_{j} \int_{t}^{s} Y_{j}(u) d u & =y_{j}+Z_{j}\left(\lambda_{j} s\right)-Z_{j}\left(\lambda_{j} t\right)-Y_{j}(s) \\
& \leq y_{j}+Z_{j}\left(\lambda_{j} s\right)-Z_{j}\left(\lambda_{j} t\right) \\
& ={ }^{\mathcal{L}} y_{j}+Z_{j}\left(\lambda_{j}(s-t)\right),
\end{aligned}
$$

since $Y_{j}(s) \geq 0$ when $y_{j}=Y_{j}(t) \geq 0$, and " $={ }^{\mathcal{L}}$ " denotes equality in law. Recall that we have defined $Z_{j}(0)=0$. We thus have, using Equation (2.15) in the last step, that

$$
\begin{aligned}
\mathbb{E}\left[\exp \left(\xi_{j} \int_{t}^{s} Y_{j}(u) d u\right)\right] & \leq \exp \left(\frac{\xi_{j}}{\lambda_{j}} y_{j}\right) \mathbb{E}\left[\exp \left(\frac{\xi_{j}}{\lambda_{j}} Z_{j}\left(\lambda_{j}(s-t)\right)\right)\right] \\
& =\exp \left(\frac{\xi_{j}}{\lambda j} y_{j}+\lambda_{j} \int_{0+}^{\infty}\left\{\exp \left(\frac{\xi_{j} z}{\lambda j}\right)-1\right\} l_{j}(d z)(s-t)\right) .
\end{aligned}
$$

Lemma 3.2 Assume Condition 2.1 holds for some positive constant $c_{j}$. Then

$$
\mathbb{E}\left[\exp \left(c_{j} Y_{j}(s)\right)\right] \leq \exp \left(c_{j} y_{j}+\lambda_{j} \int_{0+}^{\infty}\left\{\exp \left(c_{j} z\right)-1\right\} l_{j}(d z)(s-t)\right)
$$

Proof. We see from Equation (2.1) that

$$
\begin{aligned}
c_{j} Y_{j}(s) & \leq c_{j} y_{j}+c_{j} Z_{j}\left(\lambda_{j} s\right)-c_{j} Z_{j}\left(\lambda_{j} t\right) \\
& ={ }^{\mathcal{L}} c_{j} y_{j}+c_{j} Z_{j}\left(\lambda_{j}(s-t)\right) .
\end{aligned}
$$

The result follows from Equation (2.15).

Lemma 3.3 Assume Condition 2.1 holds with

$$
c_{j}=\frac{2 \theta\left(\left|\beta_{1}\right|+\theta\right) \omega_{1, j}+2 \theta\left(\left|\beta_{2}\right|+\theta\right) \omega_{2, j}}{\lambda_{j}}, \quad j=1, \ldots, m,
$$

for some $\theta>0$. Then

$$
\begin{aligned}
& \sup _{\pi \in \mathcal{A}_{t}} \mathbb{E}^{t, w, y}\left[\left(W^{\pi}(s)\right)^{\theta}\right] \\
& \leq w^{\theta} \exp \left(2 \theta \sum_{j=1}^{m} \frac{\left(\left|\beta_{1}\right|+\theta\right) \omega_{1, j}+\left(\left|\beta_{2}\right|+\theta\right) \omega_{2, j}}{\lambda_{j}} y_{j}+C(\theta)(s-t)\right),
\end{aligned}
$$

where

$$
\begin{aligned}
C(\theta)=\theta & \left(\left|\mu_{1}-r\right|+\left|\mu_{2}-r\right|+r\right) \\
& +\frac{1}{2} \sum_{j=1}^{m} \lambda_{j} \int_{0+}^{\infty}\left(\exp \left(2 \theta \frac{\left(\left|\beta_{1}\right|+\theta\right) \omega_{1, j}+\left(\left|\beta_{2}\right|+\theta\right) \omega_{2, j}}{\lambda_{j}} z-1\right)\right) l_{j}(d z) .
\end{aligned}
$$


Proof. We have by Equation (2.14) and Itô's formula that

$$
\begin{aligned}
W^{\pi}(s)=w & \exp \left(\int_{t}^{s} \alpha\left(u, \sigma_{1}(u), \sigma_{2}(u)\right) d u\right. \\
& \left.+\int_{s}^{t} \pi_{1}(u) \sqrt{\sigma_{1}(u)} d B_{1}(u)+\int_{s}^{t} \pi_{2}(u) \sqrt{\sigma_{2}(u)} d B_{2}(u)\right),
\end{aligned}
$$

where

$$
\begin{gathered}
\alpha\left(u, \sigma_{1}, \sigma_{2}\right)=\pi_{1}(u)\left(\mu_{1}+\beta_{1} \sigma_{1}-r\right)+\pi_{2}(u)\left(\mu_{2}+\beta_{2} \sigma_{2}-r\right) \\
+r-\frac{1}{2}\left(\pi_{1}(u)\right)^{2} \sigma_{1}-\frac{1}{2}\left(\pi_{2}(u)\right)^{2} \sigma_{2} .
\end{gathered}
$$

Define

$$
\begin{aligned}
X(s)=\exp & \left(\int_{t}^{s} 2 \theta \pi_{1}(u) \sqrt{\sigma_{1}(u)} d B_{1}(u)+\int_{t}^{s} 2 \theta \pi_{2}(u) \sqrt{\sigma_{2}(u)} d B_{2}(u)\right. \\
& \left.-\frac{1}{2} \int_{t}^{s}(2 \theta)^{2}\left(\pi_{1}(u)\right)^{2} \sigma_{1}(u) d u-\frac{1}{2} \int_{t}^{s}(2 \theta)^{2}\left(\pi_{2}(u)\right)^{2} \sigma_{2}(u) d u\right) .
\end{aligned}
$$

Since the processes $Y_{j}(s)$ are right-continuous we have that $\sigma_{i}(s), i=1,2$, are right-continuous. Due to the exponential integrability conditions on $Y_{j}$ we have that

$$
\mathbb{E}\left[\exp \left(\frac{1}{2} \int_{0}^{T} \sigma_{1}(t)+\sigma_{2}(t) d t\right)\right]<\infty .
$$

This implies that $\int_{0}^{t} \pi_{i}(u) \sqrt{\sigma_{i}(u)} d B_{i}(u), i=1,2$, are well-defined continuous martingales. Then $X(s)$ is a martingale by Novikov's condition (see $[19, \mathrm{p}$. $140])$, and $\mathbb{E}[X(s)]=1$. Lemma 3.1 with $\xi_{j}=2 \theta^{2}\left(\omega_{1, j}+\omega_{2, j}\right), j=1, \ldots, m$, gives

$$
\begin{aligned}
& \mathbb{E}\left[e^{\frac{1}{2} \int_{t}^{T}(2 \theta)^{2}\left(\pi_{1}(u)\right)^{2} \sigma_{1}(u) d u+\frac{1}{2} \int_{t}^{T}(2 \theta)^{2}\left(\pi_{2}(u)\right)^{2} \sigma_{2}(u) d u}\right] \\
& \leq \mathbb{E}\left[e^{2 \theta^{2} \int_{t}^{T} \sigma_{1}(u) d u+2 \theta^{2} \int_{t}^{T} \sigma_{2}(u) d u}\right] \\
& =\prod_{j=1}^{m} \mathbb{E}\left[e^{2 \theta^{2}\left(\omega_{1, j}+\omega_{2, j}\right) \int_{t}^{T} Y_{j}(u) d u}\right]<\infty .
\end{aligned}
$$

Hence, by Hölder's inequality and using that $\pi_{i} \in[0,1], i=1,2$,

$$
\begin{aligned}
& \mathbb{E}\left[\left(W^{\pi}(s)\right)^{\theta}\right] \\
& =w^{\theta} \mathbb{E}\left[\operatorname { e x p } \left(\theta \int_{t}^{s} \alpha\left(u, \sigma_{1}(u), \sigma_{2}(u)\right) d u\right.\right. \\
& \left.\left.\quad+\theta \int_{t}^{s} \pi_{1}(u) \sqrt{\sigma_{1}(u)} d B_{1}(u)+\theta \int_{t}^{s} \pi_{2}(u) \sqrt{\sigma_{2}(u)} d B_{2}(u)\right)\right]
\end{aligned}
$$




$$
\begin{aligned}
=w^{\theta} \mathbb{E} & {\left[\operatorname { e x p } \left(\theta \int_{t}^{s} \alpha\left(u, \sigma_{1}(u), \sigma_{2}(u)\right) d u\right.\right.} \\
& \left.\left.+\theta^{2} \int_{t}^{s}\left(\pi_{1}(u)\right)^{2} \sigma_{1}(u)+\left(\pi_{2}(u)\right)^{2} \sigma_{2}(u) d u\right) X(s)^{\frac{1}{2}}\right] \\
\leq w^{\theta} \mathbb{E}\left[\operatorname { e x p } \left(\int_{t}^{s} 2 \theta \alpha\left(u, \sigma_{1}(u), \sigma_{2}(u)\right) d u\right.\right. & \\
& \left.\left.+2 \theta^{2} \int_{t}^{s}\left(\pi_{1}(u)\right)^{2} \sigma_{1}(u)+\left(\pi_{2}(u)\right)^{2} \sigma_{2}(u) d u\right)\right]^{\frac{1}{2}} \times \mathbb{E}[X(s)]^{\frac{1}{2}} \\
\leq w^{\theta} e^{(s-t) \theta\left(\left|\mu_{1}-r\right|+\left|\mu_{2}-r\right|+r\right)} & \times \mathbb{E}^{s}\left[\exp \left(2 \theta\left(\left|\beta_{1}\right|+\theta\right) \int_{t}^{s} \sigma_{1}(u) d u+2 \theta\left(\left|\beta_{2}\right|+\theta\right) \int_{t}^{s} \sigma_{2}(u) d u\right)\right]^{\frac{1}{2}} \\
=w^{\theta} e^{(s-t) \theta\left(\left|\mu_{1}-r\right|+\left|\mu_{2}-r\right|+r\right)} & \times \prod_{j=1}^{m}\left[\mathbb{E}\left[\exp \left(2 \theta\left(\left(\left|\beta_{1}\right|+\theta\right) \omega_{1, j}+\left(\left|\beta_{2}\right|+\theta\right) \omega_{2, j}\right) \int_{t}^{s} Y_{j}(u) d u\right)\right]^{\frac{1}{2}}\right.
\end{aligned}
$$

Applying Lemma 3.1 with

$$
\xi_{j}=2 \theta\left(\left|\beta_{1}\right|+\theta\right) \omega_{1, j}+2 \theta\left(\left|\beta_{2}\right|+\theta\right) \omega_{2, j}, \quad j=1, \ldots, m,
$$

proves the result.

We now use the result above results to show that the value function of our control problem is well-defined.

Proposition 3.1 Assume Condition 2.1 holds with $c_{j}$ defined as in Lemma 3.3. Then

$$
\begin{aligned}
U(0) \leq & V(t, w, y) \\
\leq k & \left(1+w^{\theta} \exp \left(2 \theta \sum_{j=1}^{m} \frac{\left(\left|\beta_{1}\right|+\theta\right) \omega_{1, j}+\left(\left|\beta_{2}\right|+\theta\right) \omega_{2, j}}{\lambda_{j}} y_{j}\right.\right. \\
& +C(\theta)(T-t))),
\end{aligned}
$$

where $C(\theta)$ is defined as in Lemma 3.3 and $k>0$.

Proof. We have that $U(w) \geq U(0)$ since $U$ is non-decreasing. This gives that $\mathbb{E}\left[U\left(W^{\pi}(T)\right)\right] \geq U(0)$, for $\pi \in \mathcal{A}_{t}$, which implies that $V(t, w, y) \geq U(0)$. The upper bound follows from the sublinear growth condition of $U$ and Lemma 3.3:

$$
\begin{aligned}
V(t, w, y)= & \sup _{\pi \in \mathcal{A}_{t}} \mathbb{E}\left[U\left(W^{\pi}(T)\right)\right] \leq k\left(1+\sup _{\pi \in \mathcal{A}_{t}} \mathbb{E}\left[U\left(W^{\pi}(T)\right)\right]^{\theta}\right) \\
\leq k & \left(1+w^{\theta} \exp \left(2 \theta \sum_{j=1}^{m} \frac{\left(\left|\beta_{1}\right|+\theta\right) \omega_{1, j}+\left(\left|\beta_{2}\right|+\theta\right) \omega_{2, j}}{\lambda_{j}} y_{j}\right.\right. \\
& +C(\theta)(T-t))) .
\end{aligned}
$$


From now on we assume that Condition 2.1 holds with

$$
c_{j}=\frac{2 \theta\left(\left|\beta_{1}\right|+\theta\right) \omega_{1, j}+2 \theta\left(\left|\beta_{2}\right|+\theta\right) \omega_{2, j}}{\lambda_{j}}, \quad j=1, \ldots, m .
$$

This ensures that the value function is well-defined.

\section{A verification theorem}

We state and prove the following verification theorem for our stochastic control problem.

Theorem 4.1 Assume that

$$
v(t, w, y) \in C^{1,2,1}\left([0, T) \times(0, \infty) \times[0, \infty)^{m}\right) \cap C([0, T] \times \bar{D})
$$

is a solution of the HJB equation (2.17) with terminal condition (2.18) and boundary condition (2.19). For $j=1, \ldots, m$, assume

$\sup _{\pi \in \mathcal{A}_{t}} \int_{0}^{T} \int_{0+}^{\infty} \mathbb{E}\left[\left|v\left(s, W^{\pi}(s), Y(s-)+z \cdot e_{j}\right)-v\left(s, W^{\pi}(s), Y(s-)\right)\right|\right] l_{j}(d z) d s<\infty$,

and

$$
\sup _{\pi \in \mathcal{A}_{t}} \int_{0}^{T} \mathbb{E}\left[\left(\pi_{i}(s)\right)^{2} \sigma_{i}(s)\left(W^{\pi}(s)\right)^{2}\left(v_{w}\left(s, W^{\pi}(s), Y(s)\right)\right)^{2}\right] d s<\infty, \quad i=1,2 .
$$

Then

$$
v(t, w, y) \geq V(t, w, y), \quad \text { for all }(t, w, y) \in[0, T] \times \bar{D} .
$$

If, in addition, there exist measurable functions $\pi_{i}^{*}(t, w, y) \in[0,1], i=1,2$, being the maximizers for the max-operator in Equation (2.17), then $\pi^{*}=\left(\pi_{1}^{*}, \pi_{2}^{*}\right)$ defines an optimal investment strategy in feedback form if Equation (2.14) admits a unique solution $W^{\pi^{*}}$ and

$$
V(t, w, y)=v(t, w, y)=\mathbb{E}^{t, w, y}\left[U\left(W^{\pi^{*}}(T)\right)\right], \text { for all }(t, w, y) \in[0, T] \times \bar{D} .
$$

The notation $C^{1,2,1}\left([0, T) \times(0, \infty) \times[0, \infty)^{m}\right)$ means twice continuously differentiable in $w$ on $(0, \infty)$ and once continuously differentiable in $t, y$ on $[0, T) \times$ $[0, \infty)^{m}$ with continuous extensions of the derivatives to $t=0$ and $y_{j}=0$, $j=1, \ldots, m$.

Proof. Let $(t, w, y) \in[0, T) \times D$ and $\pi \in \mathcal{A}_{t}$, and introduce the operator

$$
\begin{aligned}
\mathcal{M}^{\pi} v:= & \left(\pi_{1}\left(\mu_{1}+\beta_{1} \sigma_{1}-r\right)+\pi_{2}\left(\mu_{2}+\beta_{2} \sigma_{2}-r\right)\right) w v_{w} \\
& +\frac{1}{2}\left(\pi_{1}^{2} \sigma_{1}+\pi_{2}^{2} \sigma_{2}\right) w^{2} v_{w w}+r w v_{w}-\sum_{j=1}^{m} \lambda_{j} y_{j} v_{y_{j}} .
\end{aligned}
$$


Itô's formula gives that

$$
\begin{aligned}
& v\left(s, W^{\pi}(s), Y(s)\right) \\
& =v(t, w, y)+\int_{t}^{s}\left\{v_{t}\left(u, W^{\pi}(u), Y(u-)\right)+\mathcal{M}^{\pi} v\left(u, W^{\pi}(u), Y(u)\right)\right\} d u \\
& \quad+\int_{t}^{s} \pi_{1}(u) \sqrt{\sigma_{1}(u)} W^{\pi}(u) v_{w}\left(u, W^{\pi}(u), Y(u)\right) d B_{1}(u) \\
& \quad+\int_{t}^{s} \pi_{2}(u) \sqrt{\sigma_{2}(u)} W^{\pi}(u) v_{w}\left(u, W^{\pi}(u), Y(u)\right) d B_{2}(u) \\
& \quad+\sum_{j=1}^{m} \int_{t}^{s} \int_{0+}^{\infty}\left(v\left(u, W^{\pi}(u), Y(u-)+z \cdot e_{j}\right)-v\left(u, W^{\pi}(u), Y(u-)\right)\right) N_{j}\left(\lambda_{j} d u, d z\right),
\end{aligned}
$$

where $N_{j}$ is the Poisson random measure coming from the Lévy-Khintchine representation of the subordinator $Z_{j}$. We know from the assumptions that the Itô integrals are martingales and that the integrals with respect to $N_{j}$ are semimartingales. This gives us that

$$
\begin{aligned}
& \mathbb{E}\left[v\left(s, W^{\pi}(s), Y(s)\right)\right] \\
& =v(t, w, y)+\mathbb{E}\left[\int_{t}^{s}\left(v_{t}+\mathcal{L}^{\pi} v\right)\left(u, W^{\pi}(u), Y(u)\right) d u\right] \\
& \leq v(t, w, y)+\mathbb{E}\left[\int_{t}^{s}\left(v_{t}+\max _{\substack{\pi_{i} \in[0,1], i=1,2, \pi_{1}+\pi_{2} \leq 1}} \mathcal{L}^{\pi} v\right)\left(u, W^{\pi}(u), Y(u)\right) d u\right] \\
& =v(t, w, y),
\end{aligned}
$$

where

$$
\mathcal{L}^{\pi} v:=\mathcal{M}^{\pi} v+\sum_{j=1}^{m} \lambda_{j} \int_{0+}^{\infty}\left(v\left(t, w, y+z \cdot e_{j}\right)-v(t, w, y)\right) l_{j}(d z) .
$$

We now get that

$$
v(t, w, y) \geq \mathbb{E}\left[U\left(W^{\pi}(T)\right)\right],
$$

for all $\pi \in \mathcal{A}_{t}$, by putting $s=T$ and invoking the terminal condition for $v$. The first conclusion in the theorem now follows by observing that the result holds for $t=T$ and $w=0$.

We prove the second part by observing that since for each $i=1,2, \pi_{i}^{*}(t, w, y)$ is assumed to be a measurable function, we have that $\pi_{i}^{*}(s, W(s), Y(s))$ is $\mathcal{F}_{s^{-}}$ measurable for $t \leq s \leq T$. This, together with the assumptions that $\pi_{i}^{*} \in[0,1]$ and the existence of a unique solution $W^{\pi^{*}}$ of Equation (2.14), implies that $\pi^{*}(s, W(s), Y(s))$ is an admissible control. Moreover, since $\pi^{*}$ is a maximizer,

$$
\max _{\substack{\pi_{i} \in[0,1], i=1,2, \pi_{1}+\pi_{2} \leq 1}} \mathcal{L}^{\pi} v=\mathcal{L}^{\pi^{*}} v
$$


The above calculations using Itô's formula go through with equality by letting $\pi=\pi^{*}$. Hence,

$$
v(t, w, y)=\mathbb{E}\left[U\left(W^{\pi^{*}}(T)\right)\right] \leq V(t, w, y) .
$$

Together with the first part of the theorem, this yields

$$
v(t, w, y)=V(t, w, y)=\mathbb{E}\left[U\left(W^{\pi^{*}}(T)\right)\right],
$$

for $(t, w, y) \in[0, T] \times \bar{D}$, since the equality obviously holds for $t=T$ and $w=0$.

\section{Explicit solution}

In this section we construct and verify an explicit solution to the control problem (2.16), as well as an explicit optimal control $\pi^{*}$, when the utility function is of the form

$$
U(w)=\gamma^{-1} w^{\gamma}, \quad \gamma \in(0,1) .
$$

\subsection{Reduction of the HJB equation}

In this subsection we reduce the HJB equation (2.17) to a first-order integrodifferential equation by making a conjecture that the value function $v$ has a certain form.

We conjecture that the value function has the form

$$
v(t, w, y)=\gamma^{-1} w^{\gamma} h(t, y), \quad(t, w, y) \in[0, T] \times \bar{D},
$$

for some function $h(t, y)$. We define the function $\Pi:[0, \infty) \times[0, \infty) \rightarrow \mathbb{R}$ as

$$
\begin{gathered}
\Pi\left(\sigma_{1}, \sigma_{2}\right)=\max _{\substack{\pi_{i} \in[0,1], i=1,2 \\
\pi_{1}+\pi_{2} \leq 1}}\left\{\pi_{1}\left(\mu_{1}+\beta_{1} \sigma_{1}-r\right)+\pi_{2}\left(\mu_{2}+\beta_{2} \sigma_{2}-r\right)\right. \\
\left.-\quad \frac{1}{2}\left(\pi_{1}^{2} \sigma_{1}+\pi_{2}^{2} \sigma_{2}\right)(1-\gamma)\right\}+r .
\end{gathered}
$$

If we insert the conjectured value function into the HJB equation (2.17) we get a first-order integro-differential equation for $h$ as

$$
\begin{aligned}
h_{t}(t, y)=- & \gamma \Pi\left(\sigma_{1}, \sigma_{2}\right) h(t, y)+\sum_{j=1}^{m} \lambda_{j} y_{j} h_{y_{j}}(t, y) \\
& \quad-\sum_{j=1}^{m} \lambda_{j} \int_{0+}^{\infty}\left(h\left(t, y+z \cdot e_{j}\right)-h(t, y)\right) l_{j}(d z),
\end{aligned}
$$

where $(t, y) \in[0, T) \times[0, \infty)^{m}$. The terminal condition becomes

$$
h(T, y)=1, \quad \forall y \in[0, \infty)^{m},
$$

since $v(T, w, y)=U(w)=\gamma^{-1} w^{\gamma}$.

For our purposes, we will need $\Pi$ to be continuously differentiable. 


\subsection{Continuous differentiability of $\Pi$}

Here we prove that $\Pi$ is continuously differentiable. We also obtain candidates for optimal fractions of wealth.

A first-order condition for an interior optimum of $\Pi\left(\sigma_{1}, \sigma_{2}\right)$ is

$$
\left(\mu_{i}+\beta_{i} \sigma_{i}-r\right)-\pi_{i} \sigma_{i}(1-\gamma)=0, \quad i=1,2 .
$$

If we denote the interior optimum by $\bar{\pi}_{i}=\bar{\pi}_{i}\left(\sigma_{i}\right), i=1,2$, then we have

$$
\bar{\pi}_{i}\left(\sigma_{i}\right)=\frac{1}{1-\gamma}\left(\frac{\mu_{i}-r}{\sigma_{i}}+\beta_{i}\right), \quad i=1,2 .
$$

We get from inspection that Equation (5.1) is continuous and differentiable whenever $\bar{\pi}_{i} \in(0,1), i=1,2, \bar{\pi}_{1}+\bar{\pi}_{2}<1$. Elementary calculus now gives that $\pi_{i}^{*}=0$, when $\bar{\pi}_{i} \leq 0, i=1,2$, and that for $\bar{\pi}_{1}+\bar{\pi}_{2} \geq 1$, the vector of optimal fractions of wealth is of the form

$$
\left(\pi_{1}^{*}, \pi_{2}^{*}\right)=(\breve{\pi}, 1-\breve{\pi}), \quad \breve{\pi} \in[0,1] .
$$

In the latter case, Equation (5.1) alters to

$$
\begin{gathered}
\breve{\Pi}\left(\sigma_{1}, \sigma_{2}\right)=\max _{\pi \in[0,1]}\left\{\pi\left(\mu_{1}+\beta_{1} \sigma_{1}-r\right)+(1-\pi)\left(\mu_{2}+\beta_{2} \sigma_{2}-r\right)\right. \\
\left.-\frac{1}{2}\left(\pi^{2} \sigma_{1}+(1-\pi)^{2} \sigma_{2}\right)(1-\gamma)\right\}+r .
\end{gathered}
$$

Here the first-order condition for an interior optimum is

$$
\left(\mu_{1}+\beta_{1} \sigma_{1}-r\right)-\left(\mu_{2}+\beta_{2} \sigma_{2}-r\right)-\left(\bar{\pi} \sigma_{1}-(1-\bar{\pi}) \sigma_{2}\right)(1-\gamma)=0 .
$$

In the name of consequence, we denote the interior optimum by $\bar{\pi}=\bar{\pi}\left(\sigma_{1}, \sigma_{2}\right)$. This gives that

$$
\begin{aligned}
& \bar{\pi}\left(\sigma_{1}, \sigma_{2}\right) \\
& =\frac{1}{(1-\gamma)}\left(\frac{\left(\mu_{1}+\beta_{1} \sigma_{1}-r\right)-\left(\mu_{2}+\beta_{2} \sigma_{2}-r\right)}{\left(\sigma_{1}+\sigma_{2}\right)}\right)+\frac{\sigma_{2}}{\left(\sigma_{1}+\sigma_{2}\right)},
\end{aligned}
$$

and we easily see that Equation (5.3) is continuous and differentiable on $\bar{\pi} \in$ $(0,1)$.

We will now prove that $\lim _{\sigma \rightarrow \hat{\sigma}_{i}} \Pi_{\sigma_{i}}^{\prime}\left(\hat{\sigma}_{1}, \hat{\sigma}_{2}\right)=\lim _{\sigma \rightarrow \sigma_{i}} \breve{\Pi}_{\sigma_{i}}^{\prime}\left(\hat{\sigma}_{1}, \hat{\sigma}_{2}\right), i=1,2$, for $\left(\hat{\sigma}_{1}, \hat{\sigma}_{2}\right)$ such that $\bar{\pi}_{1}+\bar{\pi}_{2}=1$. We prove the result for the derivative taken in $\sigma_{1}$, the result for $\sigma_{2}$ being analogous. The key to this result is to observe that when $\bar{\pi}_{1}+\bar{\pi}_{2}=1$,

$$
\begin{aligned}
\bar{\pi}_{1} & =\frac{1}{1-\gamma}\left(\frac{\mu_{1}-r}{\sigma_{1}}+\beta_{1}\right) \\
& =\frac{1}{(1-\gamma)}\left(\frac{\left(\mu_{1}+\beta_{1} \sigma_{1}-r\right)-\left(\mu_{2}+\beta_{2} \sigma_{2}-r\right)}{\left(\sigma_{1}+\sigma_{2}\right)}\right)+\frac{\sigma_{2}}{\left(\sigma_{1}+\sigma_{2}\right)} .
\end{aligned}
$$


For notational simplicity, set $\tau_{i}=\left(\mu_{i}+\beta_{i} \sigma_{i}-r\right), i=1,2$. Calculations show that

$$
\begin{aligned}
\Pi_{\sigma_{1}}^{\prime}= & \frac{1}{2(1-\gamma)}\left(\frac{2\left(\tau_{1}-\tau_{2}\right) \beta_{1}\left(\sigma_{1}+\sigma_{2}\right)-\left(\tau_{1}-\tau_{2}\right)^{2}}{\left(\sigma_{1}+\sigma_{2}\right)^{2}}\right) \\
& +\sigma_{2}\left(\frac{\beta_{1}\left(\sigma_{1}+\sigma_{2}\right)-\left(\tau_{1}-\tau_{2}\right)}{\left(\sigma_{1}+\sigma_{2}\right)^{2}}\right)-\frac{(1-\gamma) \sigma_{2}^{2}}{2\left(\sigma_{1}+\sigma_{2}\right)^{2}} \\
= & \bar{\pi}_{1} \beta_{1}-\frac{\left(\tau_{1}-\tau_{2}\right)^{2}}{2(1-\gamma)\left(\sigma_{1}+\sigma_{2}\right)^{2}}-\frac{\sigma_{2}\left(\tau_{1}-\tau_{2}\right)}{\left(\sigma_{1}+\sigma_{2}\right)^{2}}-\frac{(1-\gamma) \sigma_{2}^{2}}{2\left(\sigma_{1}+\sigma_{2}\right)^{2}} \\
= & \bar{\pi}_{1} \beta_{1}-\frac{(1-\gamma)}{2} \bar{\pi}_{1}^{2} .
\end{aligned}
$$

But we also have that

$$
\breve{\Pi}_{\sigma_{1}}^{\prime}=\frac{1}{2(1-\gamma)}\left(\frac{2 \tau_{1} \beta_{1} \sigma_{1}-\tau_{1}^{2}}{\sigma_{1}^{2}}\right)=\bar{\pi}_{1} \beta_{1}-\frac{(1-\gamma)}{2} \bar{\pi}_{1}^{2} .
$$

The proof that $\Pi$ is continuously differentiable when $\pi_{i}=0, i=1,2$, is similar to the result above and we omit it.

By the results of this subsection we can now conclude that for $i=1,2$, our candidates for optimal fractions of wealth are

$$
\pi_{i}^{*}\left(\sigma_{i}\right)=\frac{1}{1-\gamma}\left(\frac{\mu_{i}-r}{\sigma_{i}}+\beta_{i}\right),
$$

whenever $\bar{\pi}_{i} \in(0,1)$ and $\bar{\pi}_{1}+\bar{\pi}_{2}<1$, and

$$
\pi_{i}^{*}=0
$$

when $\bar{\pi}_{i} \leq 0$. When $\bar{\pi}_{1}+\bar{\pi}_{2} \geq 1$, the optimal fractions of wealth are

$$
\pi_{1}^{*}\left(\sigma_{1}, \sigma_{2}\right)=\frac{1}{(1-\gamma)}\left(\frac{\left(\mu_{1}+\beta_{1} \sigma_{1}-r\right)-\left(\mu_{2}+\beta_{2} \sigma_{2}-r\right)}{\left(\sigma_{1}+\sigma_{2}\right)}\right)+\frac{\sigma_{2}}{\left(\sigma_{1}+\sigma_{2}\right)}
$$

and

$$
\pi_{2}^{*}=1-\pi_{1}^{*} .
$$

Remark 5.1 Note that we can find a constant $\alpha>0$ such that

$$
\left|\Pi\left(\sigma_{1}, \sigma_{2}\right)\right| \leq \alpha+\left|\beta_{1}\right| \sigma_{1}+\left|\beta_{2}\right| \sigma_{2} .
$$

\subsection{A Feynman-Kac formula}

In this subsection we define a Feynman-Kac formula that we verify as a classical solution to the related forward problem of Equation (5.2). 
Define the function $g(t, y)$ by

$$
g(t, y)=\mathbb{E}^{y}\left[\exp \left(\int_{0}^{t} \gamma \Pi\left(\sigma_{1}(s), \sigma_{2}(s)\right) d s\right)\right], \quad(t, y) \in[0, T] \times[0, \infty)^{m},
$$

where we denote $\sigma_{i}^{y}:=\sigma_{i}^{0, y}=\sum_{j=1}^{m} \omega_{i, j} y_{j}, i=1,2$, for $y_{j}=Y_{j}(0)$. Note that $g(0, y)=1$. We now show that $g$ is well-defined under an exponential growth hypothesis in $\sigma_{1}$ and $\sigma_{2}$.

Lemma 5.1 Assume Condition 2.1 holds with $c_{j}=\frac{\gamma}{\lambda_{j}}\left(\left|\beta_{1}\right| \omega_{1, j}+\left|\beta_{2}\right| \omega_{2, j}\right)$ for $j=1, \ldots, m$. Then

$$
g(t, y) \leq \exp \left(k t+\gamma \sum_{j=1}^{m} \frac{\left(\left|\beta_{1}\right| \omega_{1, j}+\left|\beta_{2}\right| \omega_{2, j}\right)}{\lambda_{j}} y_{j}\right),
$$

for some positive constant $k$.

Proof. From Remark 5.1 we know that

$$
\left|\Pi\left(\sigma_{1}, \sigma_{2}\right)\right| \leq \alpha+\left|\beta_{1}\right| \sigma_{1}+\left|\beta_{2}\right| \sigma_{2}
$$

for some constant $\alpha>0$. Therefore,

$$
\begin{aligned}
g(t, y) & =\mathbb{E}^{y}\left[\exp \left(\int_{0}^{t} \gamma \Pi\left(\sigma_{1}(s), \sigma_{2}(s)\right) d s\right)\right] \\
& \leq \mathbb{E}^{y}\left[\exp \left(\int_{0}^{t} \gamma \alpha+\gamma\left|\beta_{1}\right| \sigma_{1}(s)+\gamma\left|\beta_{2}\right| \sigma_{2}(s) d s\right)\right] \\
& \leq e^{\gamma \alpha t} \mathbb{E}^{y}\left[\prod_{j=1}^{m} e^{\gamma\left(\left|\beta_{1}\right| \omega_{1, j}+\left|\beta_{2}\right| \omega_{2, j}\right) \int_{0}^{t} Y_{j}^{y_{j}}(s) d s}\right] .
\end{aligned}
$$

By independence of the $Y_{j}, j=1, \ldots, m$, we get by Lemma 3.1 that

$$
\begin{aligned}
g(t, y) \leq e^{\gamma a t} \mathbb{E}^{y}\left[\prod_{j=1}^{m} e^{\gamma\left(\left|\beta_{1}\right| \omega_{1, j}+\left|\beta_{2}\right| \omega_{2, j}\right) \int_{0}^{t} Y_{j}^{y_{j}}(s) d s}\right] \\
\leq e^{\gamma a t} \prod_{j=1}^{m} \exp \left(\frac{\gamma\left(\left|\beta_{1}\right| \omega_{1, j}+\left|\beta_{2}\right| \omega_{2, j}\right)}{\lambda_{j}} y_{j}\right. \\
\left.\quad+t \lambda_{j} \int_{0+}^{\infty}\left(\exp \left(\frac{\gamma\left(\left|\beta_{1}\right| \omega_{1, j}+\left|\beta_{2}\right| \omega_{2, j}\right)}{\lambda_{j}} z\right)-1\right) l_{j}(d z)\right)
\end{aligned}
$$

Hence, there exists a positive constant $k$ such that

$$
g(t, y) \leq e^{k t+\gamma b \sum_{j=1}^{m} \frac{\gamma\left(\left|\beta_{1}\right| \omega_{1, j}+\left|\beta_{2}\right| \omega_{2, j}\right)}{\lambda_{j}} y_{j}},
$$

and we are done.

We now aim to show that $g$ is continuously differentiable in $y$. 
Lemma 5.2 Assume Condition 2.1 holds with $c_{j}=\frac{\gamma}{\lambda_{j}}\left(\left|\beta_{1}\right| \omega_{1, j}+\left|\beta_{2}\right| \omega_{2, j}\right)$, $j=1, \ldots, m$. Then $g \in C^{0,1}\left(([0, T]) \times[0, \infty)^{m}\right)$; that is, $g(t, \cdot)$ is continuous for all $t \in[0, T]$ and $g(\cdot, y)$ is once continuously differentiable for all $y \in[0, \infty)^{m}$.

Proof. We will use the dominated convergence theorem to prove that we can interchange expectation and differentiation. Sufficiently general conditions for us to do this are contained in Theorem 2.27 in [10], which essentially says that we need to bound the derivative by an integrable function independent of $y$.

Let $(t, y) \in[0, T] \times \mathbb{R}_{+}^{m}$ and set

$$
F(t, y)=e^{\int_{0}^{t} \gamma \Pi\left(\sigma_{1}^{y}(s), \sigma_{2}^{y}(s)\right) d s} .
$$

For each $j=1, \ldots, m$, we have

$$
\frac{\partial F(t, y)}{\partial y_{j}}=\left(\frac{\partial}{\partial y_{j}} \int_{0}^{t} \gamma \Pi\left(\sigma_{1}^{y}(s), \sigma_{2}^{y}(s)\right) d s\right) e^{\int_{0}^{t} \gamma \Pi\left(\sigma_{1}^{y}(s), \sigma_{2}^{y}(s)\right) d s} .
$$

Since $\Pi$ is continuously differentiable and $\Pi^{\prime}$ is bounded

$$
\gamma \Pi^{\prime}\left(\sigma_{1}(s), \sigma_{2}(s)\right) \frac{\partial \sigma^{y}(s)}{\partial y_{j}}=\gamma \Pi^{\prime}\left(\sigma_{1}^{y}(s), \sigma_{2}^{y}(s)\right) \omega_{j} e^{-\lambda_{j} s} \leq c e^{-\lambda_{j} s}
$$

for some strictly positive constant $c$. Theorem $2.27(\mathrm{~b})$ in [10] now gives that

$$
\frac{\partial F(t, y)}{\partial y_{j}}=\left(\int_{0}^{t} \gamma \Pi^{\prime}\left(\sigma_{1}^{y}(s), \sigma_{2}^{y}(s)\right) e^{-\lambda_{j} s} d s\right) e^{\int_{0}^{t} \gamma \Pi\left(\sigma_{1}^{y}(s), \sigma_{2}^{y}(s)\right) d s} .
$$

From the assumptions we have that

$$
\begin{aligned}
\left|\frac{\partial F(t, y)}{\partial y_{j}}\right| & \leq\left(c \int_{0}^{t} e^{-\lambda_{j} s} d s\right) e^{\int_{0}^{t} \gamma \Pi\left(\left(\sigma_{1}^{y}(s), \sigma_{2}^{y}(s)\right)\right) d s} \\
& \leq \frac{c}{\lambda_{j}} e^{\gamma \alpha T+\gamma\left|\beta_{1}\right| \int_{0}^{t} \sigma_{1}^{y}(s) d s+\gamma\left|\beta_{2}\right| \int_{0}^{t} \sigma_{2}^{y}(s) d s},
\end{aligned}
$$

where we once again can apply Lemma 3.1 to get that $\left|\partial F(t, y) / \partial y_{j}\right|$ is finite. Furthermore, with the aid of its proof, we can withdraw that on a compact set with $y$ in its interior, $\left|\partial F(t, y) / \partial y_{j}\right|$ is uniformly bounded in $y$ by the random variable

$$
\exp \left(\frac{\gamma\left(\left|\beta_{1}\right| \omega_{1, j}+\left|\beta_{2}\right| \omega_{2, j}\right)}{\lambda_{j}} Z_{j}\left(\lambda_{j} t\right)\right),
$$

which is integrable by Condition 2.1. Once again, 2.27(b) in [10] can be applied to show that $g(t, y)=\mathbb{E}[F(t, y)]$ is differentiable in $y$. But differentiability is a local notion. Hence the result is independent of the choice of compact set, and we conclude that

$$
\frac{\partial g(t, y)}{\partial y_{j}}=\mathbb{E}\left[\frac{\partial F(t, y)}{\partial y_{j}}\right], \quad \forall y \in \mathbb{R}_{+}^{m}, \quad j=1, \ldots, m .
$$


We also have that $y \mapsto \partial F(t, y) / \partial y_{j}$ is continuous since $y \mapsto\left(\sigma_{1}^{y}(s), \sigma_{2}^{y}(s)\right)$, $\left(\sigma_{1}^{y}(s), \sigma_{2}^{y}(s)\right) \mapsto \Pi\left(\sigma_{1}^{y}(s), \sigma_{2}^{y}(s)\right)$, and $\left(\sigma_{1}^{y}(s), \sigma_{2}^{y}(s)\right) \mapsto \Pi^{\prime}\left(\sigma_{1}^{y}(s), \sigma_{2}^{y}(s)\right)$ are all continuous mappings. By using Theorem 2.27(a) in [10] we now get that the mapping $(t, y) \mapsto \partial g(t, y) / \partial y_{j}$ is continuous.

Lemma 5.3 Assume Condition 2.1 holds with $c_{j}=2 \frac{\gamma}{\lambda_{j}}\left(\left|\beta_{1}\right| \omega_{1, j}+\left|\beta_{2}\right| \omega_{2, j}\right)$ for $j=1, \ldots, m$. Then

$$
\sum_{j=1}^{m} \mathbb{E}\left[\int_{0}^{T} \int_{0+}^{\infty}\left|g\left(u, Y(u)+z \cdot e_{j}\right)-g(u, Y(u))\right| l_{j}(d z) d u\right]<\infty .
$$

Proof. Differentiability of $g$ and the mean value theorem give that

$$
\begin{aligned}
& \left|g\left(u, y+z \cdot e_{j}\right)-g(u, y)\right| \\
& \leq \sup _{x \in[0, z]}\left|\frac{\partial g\left(u, y+x \cdot e_{j}\right)}{\partial y_{j}}\right| z \\
& \leq k z \exp \left(\sum_{j=1}^{m} \frac{\gamma\left(\left|\beta_{1}\right| \omega_{1, j}+\left|\beta_{2}\right| \omega_{2, j}\right)}{\lambda_{j}}\left(y_{j}+Z_{j}\left(\lambda_{j} u\right)\right)\right),
\end{aligned}
$$

where $k$ is a positive constant depending only on $T$ and the parameters of the problem. Since

$$
\begin{aligned}
& \frac{\gamma\left(\left|\beta_{1}\right| \omega_{1, j}+\left|\beta_{2}\right| \omega_{2, j}\right)}{\lambda_{j}}\left(Y_{j}^{y_{j}}(u)+Z_{j}\left(\lambda_{j} u\right)\right) \\
& \leq \frac{\gamma\left(\left|\beta_{1}\right| \omega_{1, j}+\left|\beta_{2}\right| \omega_{2, j}\right)}{\lambda_{j}}\left(Z_{j}\left(\lambda_{j} u\right)+y_{j}+Z_{j}\left(\lambda_{j} u\right)\right) \\
& =\frac{\gamma\left(\left|\beta_{1}\right| \omega_{1, j}+\left|\beta_{2}\right| \omega_{2, j}\right)}{\lambda_{j}}\left(y_{j}+2 Z_{j}\left(\lambda_{j} u\right)\right),
\end{aligned}
$$

we have

$$
\begin{aligned}
& \left|g\left(u, Y(u)+z \cdot e_{j}\right)-g(u, Y(u))\right| \\
& \leq k z \exp \left(\sum_{j=1}^{m} \frac{\gamma\left(\left|\beta_{1}\right| \omega_{1, j}+\left|\beta_{2}\right| \omega_{2, j}\right)}{\lambda_{j}}\left(y_{j}+2 Z_{j}\left(\lambda_{j} u\right)\right)\right) .
\end{aligned}
$$

From the Tonelli theorem, the assumptions, and Equation (2.15) we have

$$
\begin{aligned}
& \mathbb{E}\left[\int_{0}^{T} \int_{0+}^{\infty}\left|g\left(u, Y(u)+z \cdot e_{j}\right)-g(u, Y(u))\right| l_{j}(d z) d u\right] \\
& \leq \int_{0}^{T} \mathbb{E}\left[\int_{0+}^{\infty} k z l_{j}(d z)\right. \\
& \left.\quad \times \exp \left(\sum_{j=1}^{m} \frac{\gamma\left(\left|\beta_{1}\right| \omega_{1, j}+\left|\beta_{2}\right| \omega_{2, j}\right)}{\lambda_{j}}\left(y_{j}+2 Z_{j}\left(\lambda_{j} u\right)\right)\right)\right] d u
\end{aligned}
$$




$$
\begin{aligned}
& =k \int_{0+}^{\infty} z l_{j}(d z) \int_{0}^{T} \exp \left(\sum_{j=1}^{m} \frac{\gamma\left(\left|\beta_{1}\right| \omega_{1, j}+\left|\beta_{2}\right| \omega_{2, j}\right)}{\lambda_{j}} y_{j}\right. \\
& \left.\quad+\lambda_{j} u \int_{0+}^{\infty}\left(e^{\frac{2 \gamma\left(\left|\beta_{1}\right| \omega_{1, j}+\left|\beta_{2}\right| \omega_{2, j}\right)}{\lambda_{j}} z}-1\right) l_{j}(d z)\right) d u \\
& <\infty
\end{aligned}
$$

We now show that $g(t, y)$ is a classical solution to the related forward problem of Equation (5.2).

Proposition 5.1 Assume there exists $\varepsilon>0$ such that Condition 2.1 is satisfied with $c_{j}=2 \frac{\gamma}{\lambda_{j}}\left(\left|\beta_{1}\right| \omega_{1, j}+\left|\beta_{2}\right| \omega_{2, j}\right)+\varepsilon$ for $j=1, \ldots, m$. Then $g(t, \cdot)$ belongs to the domain of the infinitesimal generator of $Y$ and

$$
\begin{aligned}
\frac{\partial g(t, y)}{\partial t}=\gamma & \Pi\left(\sigma_{1}, \sigma_{2}\right) g(t, y)-\sum_{j=1}^{m} \lambda_{j} y_{j} \frac{\partial g(t, y)}{\partial y_{j}} \\
& +\sum_{j=1}^{m} \lambda_{j} \int_{0+}^{\infty}\left(g\left(t, y+z \cdot e_{j}\right)-g(t, y)\right) l_{j}(d z)
\end{aligned}
$$

for $(t, y) \in(0, T] \times[0, \infty)^{m}$. Moreover, $\partial g(t, y) / \partial t$ is continuous, so that $g \in$ $C^{1,1}\left((0, T] \times[0, \infty)^{m}\right)$.

Proof. We begin by observing that the conditions in Lemmas 5.1, 5.2, and 5.3 are fulfilled. The first two terms on the right-hand side of Equation (5.8) are continuous since $\Pi$ is continuous and $g(t, \cdot) \in C^{1}$ by Lemma 5.2, for all $t \in[0, T]$. The integral operator is also continuous in both time and space. This can be deduced from the integrability conditions on the Lévy measures $l_{j}(d z)$ and Theorem 2.27 in [10] together with arguments similar to those of the proofs of Lemmas 5.2 and 5.3. Hence, if $g$ solves Equation (5.8) then $\partial g(t, y) / \partial t$ is continuous for $(t, y) \in(0, T) \times[0, \infty)^{m}$, and may be continuously extended to $t=T$. Thus, $g \in C^{1,1}\left((0, T] \times[0, \infty)^{m}\right)$.

Since $y \mapsto g(t, y)$ is continuously differentiable by Lemma 5.2 , we conclude from Itô's lemma that the mapping $s \mapsto g(t, Y(s))$ is a local semimartingale with dynamics

$$
\begin{aligned}
g(t, Y(s))=g & (t, y)+\sum_{j=1}^{m} \lambda_{j} \int_{0}^{s} Y_{j}(u) \frac{\partial g}{\partial y_{j}}(t, Y(u)) d u \\
& +\sum_{j=1}^{m} \int_{0}^{s} \int_{0+}^{\infty}\left(g\left(t, Y(u-)+z \cdot e_{j}\right)-g(t, Y(u-))\right) N_{j}\left(\lambda_{j} d u, d z\right),
\end{aligned}
$$

where $N_{j}$ is the Poisson random measure in the Lévy-Khintchine representation of $Z_{j}$. From Lemma 5.3 we have that

$$
\mathbb{E}\left[\int_{0}^{T} \int_{0+}^{\infty}\left|g\left(u, Y(u)+z \cdot e_{j}\right)-g(u, Y(u))\right| l_{j}(d z) d u\right]<\infty,
$$


and thus $g\left(u, Y(u)+z \cdot e_{j}\right)-g(u, Y(u)) \in \mathbf{F}^{1}, j=1, \ldots, m$ (see [13, pp. 61$62]$, for this notation). This implies that $g(t, Y(s))$ is a semimartingale, since it belongs to a subclass of proper semimartingales that [13], for simplicity, define to be semimartingales. Taking expectations on both sides and applying Fubini's theorem gives

$$
\begin{aligned}
& \frac{\mathbb{E}[g(t, Y(s))-g(t, y)]}{s} \\
& =-\sum_{j=1}^{m} \frac{\lambda_{j}}{s} \int_{0}^{s} \mathbb{E}\left[Y_{j}(u) \frac{\partial g}{\partial y_{j}}(t, Y(u))\right] d u \\
& \quad+\sum_{j=1}^{m} \frac{\lambda_{j}}{s} \int_{0}^{s} \int_{0+}^{\infty} \mathbb{E}\left[g\left(t, Y(u-)+z \cdot e_{j}\right)-g(t, Y(u-))\right] l_{j}(d z) d u .
\end{aligned}
$$

Hence, if we note that $Y$ is cádlág and $y \mapsto g(t, y)$ is continuously differentiable, by letting $s \downarrow 0$ we get that $g(t, \cdot)$ is in the domain of the infinitesimal generator of $Y$, which is denoted by $\mathcal{G}$, and

$$
\mathcal{G} g(t, y)=-\sum_{j=1}^{m} \lambda_{j} y_{j} \frac{\partial g}{\partial y_{j}}(t, y)+\sum_{j=1}^{m} \lambda_{j} \int_{0+}^{\infty}\left(g\left(t, y+z \cdot e_{j}\right)-g(t, y)\right) l_{j}(d z)
$$

Since $g(t, Y(s)) \in L^{1}(\Omega, P)$ for all $s>0$ in a neighborhood of zero, the Markov property of $Y$ together with total expectation yields

$$
\begin{aligned}
& \mathbb{E}[g(t, Y(s))] \\
& =\mathbb{E}\left[\mathbb { E } \left[e^{\left.\left.\int_{0}^{t} \gamma \Pi\left(\sigma_{1}^{Y^{y}(s)}(u), \sigma_{2}^{Y^{y}(s)}(u)\right) d u\right]\right]}\right.\right. \\
& =\mathbb{E}\left[\mathbb{E}\left[e^{\int_{0}^{t} \gamma \Pi\left(\sigma_{1}^{y}(u+s), \sigma_{2}^{y}(u+s)\right) d u} \mid \mathcal{F}_{s}\right]\right] \\
& =\mathbb{E}\left[e^{\int_{s}^{t+s} \gamma \Pi\left(\sigma_{1}^{y}(u), \sigma_{2}^{y}(u)\right) d u}\right] \\
& =\mathbb{E}\left[e^{\int_{0}^{t+s} \gamma \Pi\left(\sigma_{1}^{y}(u), \sigma_{2}^{y}(u)\right) d u} e^{-\int_{0}^{s} \gamma \Pi\left(\sigma_{1}^{y}(u), \sigma_{2}^{y}(u)\right) d u}\right] .
\end{aligned}
$$

Thus,

$$
\begin{aligned}
& \frac{\mathbb{E}[g(t, Y(s))-g(t, y)]}{s} \\
& =\frac{1}{s} \mathbb{E}\left[e^{\int_{0}^{t+s} \gamma \Pi\left(\sigma_{1}^{y}(u), \sigma_{2}^{y}(u)\right) d u} e^{-\int_{0}^{s} \gamma \Pi\left(\sigma_{1}^{y}(u), \sigma_{2}^{y}(u)\right) d u}-e^{\int_{0}^{t} \gamma \Pi\left(\sigma_{1}^{y}(u), \sigma_{2}^{y}(u)\right) d u}\right] \\
& =\frac{1}{s} \mathbb{E}\left[e^{\int_{0}^{t+s} \gamma \Pi\left(\sigma_{1}^{y}(u), \sigma_{2}^{y}(u)\right) d u} e^{-\int_{0}^{s} \gamma \Pi\left(\sigma_{1}^{y}(u), \sigma_{2}^{y}(u)\right) d u}-e^{\int_{0}^{t+s} \gamma \Pi\left(\sigma_{1}^{y}(u), \sigma_{2}^{y}(u)\right) d u}\right] \\
& \quad+\frac{1}{s}\left\{\mathbb{E}\left[e^{\int_{0}^{t+s} \gamma \Pi\left(\sigma_{1}^{y}(u), \sigma_{2}^{y}(u)\right) d u}\right]-\mathbb{E}\left[e^{\int_{0}^{t} \gamma \Pi\left(\sigma_{1}^{y}(u), \sigma_{2}^{y}(u)\right) d u}\right]\right\} \\
& =\mathbb{E}\left[e^{\int_{0}^{t+s} \gamma \Pi\left(\sigma_{1}^{y}(u), \sigma_{2}^{y}(u)\right) d u} \frac{1}{s}\left\{e^{-\int_{0}^{s} \gamma \Pi\left(\sigma_{1}^{y}(u), \sigma_{2}^{y}(u)\right) d u}-1\right\}\right] \\
& \quad+\frac{g(t+s, y)-g(t, y)}{s} .
\end{aligned}
$$


By the Fundamental Theorem of Calculus we have that

$$
\begin{gathered}
e^{\int_{0}^{t+s} \gamma \Pi\left(\sigma_{1}^{y}(u), \sigma_{2}^{y}(u)\right) d u} \frac{1}{s}\left\{e^{-\int_{0}^{s} \gamma \Pi\left(\sigma_{1}^{y}(u), \sigma_{2}^{y}(u)\right) d u}-1\right\} \\
\rightarrow-\gamma \Pi\left(\sigma_{1}^{y}, \sigma_{2}^{y}\right) e^{\int_{0}^{t} \gamma \Pi\left(\sigma_{1}^{y}(u), \sigma_{2}^{y}(u)\right) d u}, \quad s \downarrow 0 .
\end{gathered}
$$

We now need to show that we may interchange limit and integration. To do this we define the function

$$
f(s)=e^{-\int_{0}^{s} \gamma \Pi\left(\sigma_{1}^{y}(u), \sigma_{2}^{y}(u)\right) d u} .
$$

From the mean value theorem and the linear growth assumption on $\Pi$ we get that

$$
\begin{aligned}
& \frac{1}{s}|f(s)-f(0)| \\
& \leq \frac{1}{s} \sup _{u \in[0, T]}\left|f^{\prime}(u)\right| s+\sup _{u \in[0, T]} \mid \gamma \Pi\left(\sigma_{1}^{y}(u), \sigma_{2}^{y}(u)\right) e^{-\int_{0}^{s} \gamma \Pi\left(\sigma_{1}^{y}(u), \sigma_{2}^{y}(u)\right) d u \mid} \\
& \leq \gamma e^{\gamma \int_{0}^{T}\left(\alpha+\left(\left|\beta_{1}\right| \sigma_{1}^{y}(u)+\left|\beta_{2}\right| \sigma_{2}^{y}(u)\right)\right) d u} \\
& \quad \times\left(\alpha+\sup _{u \in[0, T]}\left(\left|\beta_{1}\right| \sigma_{1}^{y}(u)+\left|\beta_{2}\right| \sigma_{2}^{y}(u)\right)\right) .
\end{aligned}
$$

Since each $Z_{j}$ is a non-decreasing process,

$$
\begin{aligned}
& \sup _{u \in[0, T]}\left(\left|\beta_{1}\right| \sigma_{1}^{y}(u)+\left|\beta_{2}\right| \sigma_{2}^{y}(u)\right) \\
& \leq\left(\left|\beta_{1}\right| \sigma_{1}+\left|\beta_{2}\right| \sigma_{2}\right) \\
& \quad+\sum_{j=1}^{m}\left(\left|\beta_{1}\right| \omega_{1, j}+\left|\beta_{2}\right| \omega_{2, j}\right) Z_{j}\left(\lambda_{j} T\right),
\end{aligned}
$$

which implies

$$
\begin{aligned}
& e^{\int_{0}^{t+s} \gamma \Pi\left(\sigma_{1}^{y}(u), \sigma_{2}^{y}(u)\right) d u} \frac{1}{s}\left\{e^{-\int_{0}^{s} \gamma \Pi\left(\sigma_{1}^{y}(u), \sigma_{2}^{y}(u)\right) d u}-1\right\} \\
& \leq c e^{\gamma \int_{0}^{T}\left(\alpha+\left|\beta_{1}\right| \sigma_{1}^{y}(u)+\left|\beta_{2}\right| \sigma_{2}^{y}(u)\right) d u} \\
& +d \sum_{j=1}^{m}\left(\left|\beta_{1}\right| \omega_{1, j}+\left|\beta_{2}\right| \omega_{2, j}\right) \\
& \times e^{2 \gamma \int_{0}^{T}\left(\alpha+\left|\beta_{1}\right| \sigma_{1}^{y}(u)+\left|\beta_{2}\right| \sigma_{2}^{y}(u)\right) d u} Z_{j}\left(\lambda_{j} T\right),
\end{aligned}
$$

for some positive constants $c, d$. But by the independence of the $Y_{j}$ and Equation (2.15) we have that

$$
\begin{aligned}
& \mathbb{E}\left[c e^{\gamma \int_{0}^{T}\left(\alpha+\left|\beta_{1}\right| \sigma_{1}^{y}(u)+\left|\beta_{2}\right| \sigma_{2}^{y}(u)\right) d u}\right] \\
& \leq c \exp \left(k T+\gamma \sum_{j=1}^{m} \frac{\left|\beta_{1}\right| \omega_{1, j}+\left|\beta_{2}\right| \omega_{2, j}}{\lambda j} y_{j}\right),
\end{aligned}
$$


for some constant $k$. In addition, we can use that there exists a positive constant $k_{\varepsilon}$ such that $z \leq k_{\varepsilon} e^{\varepsilon z}$, for all $z \geq 0$, which gives that

$$
\begin{aligned}
& d \sum_{j=1}^{m}\left(\left|\beta_{1}\right| \omega_{1, j}+\left|\beta_{2}\right| \omega_{2, j}\right) \\
& \quad \times \mathbb{E}\left[e^{2 \gamma \int_{0}^{T}\left(\alpha+\left|\beta_{1}\right| \sigma_{1}^{y}(u)+\left|\beta_{2}\right| \sigma_{2}^{y}(u)\right) d u} Z_{j}\left(\lambda_{j} T\right)\right] \\
& \leq \hat{d} \sum_{j=1}^{m}\left(\left|\beta_{1}\right| \omega_{1, j}+\left|\beta_{2}\right| \omega_{2, j}\right) \\
& \quad \times \mathbb{E}\left[e e^{\left.\left(\frac{2 \gamma\left(\left|\beta_{1}\right| \omega_{1, j}+\left|\beta_{2}\right| \omega_{2, j}\right)}{\lambda_{j}}+\varepsilon\right) Z_{j}\left(\lambda_{j} T\right)\right]}\right. \\
& =\hat{d} \sum_{j=1}^{m}\left(\left|\beta_{1}\right| \omega_{1, j}+\left|\beta_{2}\right| \omega_{2, j}\right) \\
& \quad \times \exp \left(\lambda_{j} T \int_{0+}^{\infty}\left(e^{\frac{2 \gamma\left(\left|\beta_{1}\right| \omega_{1, j}+\left|\beta_{2}\right| \omega_{2, j}\right)+\lambda_{j} \varepsilon}{\lambda_{j}}}-1\right) l_{j}(d z)\right),
\end{aligned}
$$

for some constant $\hat{d}$. The last sum is finite by our integrability assumption. Therefore, by dominated convergence, we see that

$$
\begin{aligned}
& \mathbb{E}\left[e^{\int_{0}^{t+s} \gamma \Pi\left(\sigma_{1}^{y}(u), \sigma_{2}^{y}(u)\right) d u} \frac{1}{s}\left\{e^{-\int_{0}^{s} \gamma \Pi\left(\sigma_{1}^{y}(u), \sigma_{2}^{y}(u)\right) d u}-1\right\}\right] \\
& =-\gamma \Pi\left(\sigma_{1}, \sigma_{2}\right) g(t, y) .
\end{aligned}
$$

Analogously, we may show that $\partial g / \partial t$ exists. We now have that

$$
\mathcal{G} g(t, y)=-\gamma \Pi\left(\sigma_{1}, \sigma_{2}\right) g(t, y)+\frac{\partial g(t, y)}{\partial t},
$$

which concludes the proof.

Define

$$
h(t, y):=g(T-t, y)=\mathbb{E}^{y}\left[e^{\int_{0}^{T-t} \gamma \Pi\left(\sigma_{1}^{y}(s), \sigma_{2}^{y}(s)\right) d s}\right],
$$

or equivalently, by the time-homogeneity of $Y$,

$$
h(t, y)=\mathbb{E}^{y}\left[e^{\int_{t}^{T} \gamma \Pi\left(\sigma_{1}^{y}(s), \sigma_{2}^{y}(s)\right) d s}\right] .
$$

From our conjecture of the form of the value function we now have our explicit solution candidate, namely

$$
v(t, w, y)=\gamma^{-1} w^{\gamma} h(t, y)
$$

The candidate for the optimal feedback control $\pi^{*}$ is given in Subsection 5.1. In the next section we prove that Equation (5.10) coincides with the value function in Equation (2.16). 


\subsection{Explicit solution of the control problem}

We will apply the verification theorem to connect our explicit solution to the value function of the control problem. To this end, we need two integrability results.

Lemma 5.4 Assume Condition 2.1 holds with

$$
c_{j}=\frac{8 \gamma}{\lambda_{j}}\left(\left(\left|\beta_{1}\right|+4 \gamma\right) \omega_{1, j}+\left(\left|\beta_{2}\right|+4 \gamma\right) \omega_{2, j}\right), \quad j=1, \ldots, m
$$

Then

$$
\int_{0}^{T} \mathbb{E}\left[\left(\pi_{i}(s)\right)^{2} \sigma_{i}(s)\left(W^{\pi}(s)\right)^{2 \gamma} h(s, Y(s))\right] d s<\infty,
$$

for all $\pi \in \mathcal{A}_{0}, i=1,2$.

Proof. We observe that the function $h$ has the same growth as $g$. Therefore by Lemma 5.1 and $\pi_{i} \in[0,1], i=1,2$,

$$
\begin{aligned}
& \int_{0}^{T} \mathbb{E}\left[\left(W^{\pi}(s)\right)^{2}\left(\left(\pi_{1}(s)\right)^{2} \sigma_{1}(s)+\left(\pi_{2}(s)\right)^{2} \sigma_{2}(s)\right)\right. \\
& \left.\quad \times\left(W^{\pi}(s)\right)^{2(\gamma-1)} h(s, Y(s))\right] d s \\
& \leq \int_{0}^{T} \mathbb{E}\left[\left(W^{\pi}(s)\right)^{2 \gamma}\left(\sigma_{1}(s)+\sigma_{2}(s)\right)\right. \\
& \left.\quad \times \exp \left(k s+\gamma \sum_{j=1}^{m} \frac{\left|\beta_{1}\right| \omega_{1, j}+\left|\beta_{2}\right| \omega_{2, j}}{\lambda j} Y_{j}(s)\right)\right] d s \\
& \leq k_{\varepsilon} e^{k T} \int_{0}^{T} \mathbb{E}\left[\left(W^{\pi}(s)\right)^{2 \gamma}\right. \\
& \left.\quad \times \exp \left((\gamma+\varepsilon) \sum_{j=1}^{m} \frac{\left|\beta_{1}\right| \omega_{1, j}+\left|\beta_{2}\right| \omega_{2, j}}{\lambda j} Y_{j}(s)\right)\right] d s
\end{aligned}
$$

where $k_{\varepsilon}$ is a positive constant such that

$$
\sigma_{1}+\sigma_{2} \leq k_{\varepsilon} e^{\varepsilon \sum_{j=1}^{m} \frac{\left|\beta_{1}\right| \omega_{1, j}+\left|\beta_{2}\right| \omega_{2, j}}{\lambda j} y_{j}}
$$

Hölder's inequality now gives

$$
\begin{aligned}
& \int_{0}^{T} \mathbb{E}\left[\left(W^{\pi}(s)\right)^{2}\left(\left(\pi_{1}(s)\right)^{2} \sigma_{1}(s)+\left(\pi_{2}(s)\right)^{2} \sigma_{2}(s)\right)\left(W^{\pi}(s)\right)^{2(\gamma-1)} h(s, Y(s))\right] \\
& \leq k_{\varepsilon} e^{k T} \int_{0}^{T} \prod_{j=1}^{m} \mathbb{E}\left[\exp \left(\frac{2(\gamma+\varepsilon)\left(\left|\beta_{1}\right| \omega_{1, j}+\left|\beta_{2}\right| \omega_{2, j}\right)}{\lambda j} Y_{j}(s)\right)\right]^{\frac{1}{2}} \\
& \times\left[\left(W^{\pi}(s)\right)^{4 \gamma}\right]^{\frac{1}{2}} d s .
\end{aligned}
$$


It is obvious that

$$
\frac{2(\gamma+\varepsilon)\left(\left|\beta_{1}\right| \omega_{1, j}+\left|\beta_{2}\right| \omega_{2, j}\right)}{\lambda j}<c_{j} \quad j=1, \ldots, m,
$$

for $\varepsilon$ sufficiently small. Hence, the integrability condition in Lemma 3.2 holds. We conclude by invoking Lemma 3.3.

Lemma 5.5 For $j=1, \ldots, m$, assume Condition 2.1 holds with

$$
c_{j}=\frac{8 \gamma}{\lambda_{j}}\left(\left(\left|\beta_{1}\right|+4 \gamma\right) \omega_{1, j}+\left(\left|\beta_{2}\right|+4 \gamma\right) \omega_{2, j}\right) .
$$

Then

$$
\int_{0}^{T} \int_{0+}^{\infty} \mathbb{E}\left[\left(W^{\pi}(u)\right)^{\gamma}\left|h\left(u, Y(u)+z \cdot e_{j}\right)-h(u, Y(u))\right|\right] l_{j}(d z) d u<\infty .
$$

Proof. Following the arguments in the proof of Lemma 5.3, and then applying Hölder's inequality with $p=4, q=4 / 3$, we get

$$
\begin{aligned}
& \int_{0}^{T} \int_{0+}^{\infty} \mathbb{E}\left[\left(W^{\pi}(u)\right)^{\gamma}\left|h\left(u, Y(u)+z \cdot e_{j}\right)-h(u, Y(u))\right|\right] l_{j}(d z) d u \\
& \leq \int_{0}^{T} \mathbb{E}\left[\left(W^{\pi}(u)\right)^{\gamma} \int_{0+}^{\infty} k_{0} z l_{j}(d z) e^{\left(\sum_{j=1}^{m} \frac{\gamma\left(\left|\beta_{1}\right| \omega_{1, j}+\left|\beta_{2}\right| \omega_{2, j}\right)}{\lambda_{j}}\left(y_{j}+2 Z_{j}\left(\lambda_{j} u\right)\right)\right)}\right] d u \\
& =k_{0} e^{k_{1} \sigma_{1}+k_{2} \sigma_{2}} \int_{0+}^{\infty} z l_{j}(d z) \\
& \quad \times \int_{0}^{T} \mathbb{E}\left[\left(W^{\pi}(u)\right)^{\gamma} e^{\left(\sum_{j=1}^{m} \frac{2 \gamma\left(\left|\beta_{1}\right| \omega_{1, j}+\left|\beta_{2}\right| \omega_{2, j}\right)}{\lambda_{j}} Z_{j}\left(\lambda_{j} u\right)\right)}\right] d u \\
& \leq k_{0} e^{k_{1} \sigma_{1}+k_{2} \sigma_{2}} \int_{0+}^{\infty} z l_{j}(d z) \\
& \quad \times \int_{0}^{T} \mathbb{E}\left[\left(W^{\pi}(u)\right)^{4 \gamma}\right]^{\frac{1}{4}} \mathbb{E}\left[e^{\left(\sum_{j=1}^{m} \frac{8 \gamma\left(\left|\beta_{1}\right| \omega_{1, j}+\left|\beta_{2}\right| \omega_{2, j}\right)}{3 \lambda_{j}} Z_{j}\left(\lambda_{j} u\right)\right)} d u\right. \\
& \leq k_{0} e^{k_{1} \sigma_{1}+k_{2} \sigma_{2}} \int_{0+}^{\infty} z l_{j}(d z) \\
& \left.\quad \times \int_{0}^{T} \mathbb{E}\left[\left(W^{\pi}(u)\right)^{4 \gamma}\right]^{\frac{3}{4}} d u \prod_{j=1}^{m} \mathbb{E}^{\left[\frac{8 \gamma\left(\left|\beta_{1}\right| \omega_{1, j}+\left|\beta_{2}\right| \omega_{2, j}\right)}{3 \lambda_{j}} Z_{j}\left(\lambda_{j} T\right)\right)}\right]^{\frac{3}{4}}
\end{aligned}
$$

for positive constants $k_{0}, k_{1}, k_{2}$. We observe that

$$
\frac{8 \gamma\left(\left|\beta_{1}\right| \omega_{1, j}+\left|\beta_{2}\right| \omega_{2, j}\right)}{3 \lambda_{j}}<c_{j},
$$

and apply Lemma 3.3 to conclude that

$$
\int_{0}^{T} \int_{0+}^{\infty} \mathbb{E}\left[\left(W^{\pi}(u)\right)^{\gamma}\left|h\left(u, Y(u)+z \cdot e_{j}\right)-h(u, Y(u))\right|\right] l_{j}(d z) d u
$$




$$
\begin{aligned}
\leq k_{0} e^{k_{1} \sigma_{1}+k_{2} \sigma_{2}} \int_{0+}^{\infty} z l_{j}(d z) \\
\quad \times \exp \left(\frac{3}{4} \sum_{j=1}^{m} \lambda_{j} T \int_{0+}^{\infty}\left(e^{\left(\frac{8 \gamma\left(\left|\beta_{1}\right| \omega_{1, j}+\left|\beta_{2}\right| \omega_{2, j}\right)}{3 \lambda_{j}} z\right)}-1\right) l_{j}(d z)\right) \\
\quad \times \int_{0}^{T} \mathbb{E}\left[\left(W^{\pi}(u)\right)^{4 \gamma}\right]^{\frac{1}{4}} d u<\infty
\end{aligned}
$$

We sum up the results in this section in the following theorem.

Theorem 5.1 Assume Condition 2.1 holds with

$$
c_{j}=\frac{8 \gamma}{\lambda_{j}}\left(\left(\left|\beta_{1}\right|+4 \gamma\right) \omega_{1, j}+\left(\left|\beta_{2}\right|+4 \gamma\right) \omega_{2, j}\right), \quad j=1, \ldots, m .
$$

Then the value function of the control problem is

$$
V(t, w, y)=\gamma^{-1} w^{\gamma} h(t, y),
$$

where $h$ is defined in (5.9). Furthermore, the optimal investment strategy is as given by Equations (5.4),..., (5.7).

Proof. We see that $V$ is well-defined under our assumption. Furthermore, the integrability condition implies by Lemma 5.1 that $h$ is well-defined, since $g$ and $h$ has the same growth. Observe that the optimal investment strategy depends only on $\left(\sigma_{1}, \sigma_{2}\right)$ and not on the level of wealth $w$. This, together with the fact that $\pi^{*} \in[0,1] \times[0,1]$, gives that there exists a unique solution $W^{\pi^{*}}$ to Equation (2.14). Hence, $\pi^{*} \in \mathcal{A}_{t}$.

Set $v(t, w, y)=\gamma^{-1} w^{\gamma} h(t, y)$, and note that the assumption in Lemmas 5.4 and 5.5 hold. We also have that the integrability conditions in Lemma 5.2 and Proposition 5.1 holds, since we may choose $\varepsilon>0$ as we like. This allows us to conclude that $v \in C([0, T] \times \bar{D})$, since $v$ is continuous in $w$ on $[0, \infty)$, and that $v$ is a classical solution of the HJB equation (2.17). We now apply Theorem 4.1, and the proof is complete.

\section{Generalizations}

In this section we state, without proofs, the most important results for the case of $n$ stocks,

$$
\pi_{i}(s) \in\left[a_{i}, b_{i}\right], \quad i=1, \ldots, n,
$$

and

$$
c \leq \sum_{i=1}^{n} \pi_{i} \leq d
$$

It can be seen that the additional difficulty in this setting is merely notational. 
The HJB equation associated to this stochastic control problem is

$$
\begin{aligned}
0=v_{t} & +\max _{\substack{\pi_{i} \in\left[a_{i}, b_{i}\right], i=1, \ldots, n, c \leq \sum_{i=1}^{n} \pi_{i} \leq d}}\left\{w v_{w} \sum_{i=1}^{n} \pi_{i}\left(\mu_{i}+\beta_{i} \sigma_{i}-r\right)+\frac{1}{2} w^{2} v_{w w} \sum_{i=1}^{n} \pi_{i}^{2} \sigma_{i}\right\} \\
& +r w v_{w}-\sum_{j=1}^{m} \lambda_{j} y_{j} v_{y_{j}}+\sum_{j=1}^{m} \lambda_{j} \int_{0}^{\infty}\left(v\left(t, w, y+z \cdot e_{j}\right)-v(t, w, y)\right) l_{j}(d z)
\end{aligned}
$$

for $(t, w, y) \in[0, T) \times D$. We still have the terminal condition

$$
V(T, w, y)=U(w), \quad \text { for all }(w, y) \in \bar{D},
$$

and the boundary condition

$$
V(t, 0, y)=U(0), \quad \text { for all }(t, y) \in[0, T] \times \mathbb{R}_{+}^{m} .
$$

The solution to this equation can be shown to be

$$
v(t, w, y)=\gamma^{-1} w^{\gamma} h(t, y)=\gamma^{-1} w^{\gamma} \mathbb{E}^{y}\left[e^{\int_{t}^{T} \gamma \Pi\left(\sigma_{1}^{y}(s), \ldots, \sigma_{n}^{y}(s)\right) d s}\right],
$$

where $\Pi$ is defined as

$$
\begin{aligned}
& \Pi\left(\sigma_{1}, \ldots, \sigma_{n}\right) \\
& =\max _{\substack{\pi_{i} \in\left[a_{i}, b_{i}\right], i=1, \ldots, n, c \leq \sum_{i=1}^{n} \pi_{i} \leq d}}\left\{\sum_{i=1}^{n} \pi_{i}\left(\mu_{i}+\beta_{i} \sigma_{i}-r\right)-\frac{1-\gamma}{2} \sum_{i=1}^{n} \pi_{i}^{2} \sigma_{i}\right\}+r .
\end{aligned}
$$

The optimal fractions of wealth are given by the parameters $\pi^{*}=\left(\pi_{1}^{*}, \ldots, \pi_{n}^{*}\right)$ that obtain $\Pi\left(\sigma_{1}, \ldots, \sigma_{n}\right)$ in Equation (6.1).

\section{$7 \quad$ Future research}

We view this paper as a starting point for more research on our $n$-asset extension of the Barndorff-Nielsen and Shephard model. Primarily, we would like to perform the statistical analysis proposed in Subsection 2.2, in order to clarify to what extent our model captures the true dependence between financial assets.

Another question of interest is portfolio optimization with the inclusion of utility of consumption. Further, it would be intriguing to consider the more general market model

$$
d S(t)=(\mu+\beta \sigma(t)) S(t) d t+\sqrt{\sigma(t)} S(t) d B(t)+\sum_{j=1}^{m} \rho_{j} \frac{S(t)}{Y_{j}(t)} d Z_{j}\left(\lambda_{j} t\right),
$$

which is a modification of a model proposed by Barndorff-Nielsen and Shephard. It allows for the so-called leverage effect to be different for the various newsprocesses, but also implies a distinction between good and bad news. A difficulty 
with this problem is that the stock prices are no longer continuous. In both these cases it ought to be feasible to solve our $n$-asset extension, once we have handled the one-asset problem.

A final issue that we aim to consider is, given our stochastic volatility market, how much higher utility an investor obtains by trading according to our optimal portfolio model compared to someone who follows the classical Merton policy.

\section{References}

[1] Barndorff-Nielsen, O.E. (1998): Processes of Normal Inverse Gaussian Type. Fin. Stoch. 2, 41-68.

[2] Barndorff-Nielsen, O. E., Shephard, N. (2001a): Modelling by Lévy Processes for Financial Econometrics. In Lévy Processes - Theory and Applications (eds O. E. Barndorff-Nielsen, T. Mikosch and S. Resnick). Boston: Birkhäuser.

[3] Barndorff-Nielsen, O. E., Shephard, N. (2001b): Non-Gaussian OrnsteinUhlenbeck-based models and some of their uses in financial economics. Journal of the Royal Statistical Society: Series B 63, 167-241 (with discussion).

[4] Benth, F. E., Karlsen, K. H., Reikvam K. (2001a): Optimal Portfolio Selection with Consumption and Non-Linear Integro-Differential Equations with Gradient Constraint: A Viscosity Solution Approach, Fin. Stoch. 5, 275-303.

[5] Benth, F. E., Karlsen, K. H., Reikvam K. (2001b): Optimal Portfolio Management Rules in a Non-Gaussian Market with Durability and Intertemporal Substitution. Fin. Stoch. 5, 447-467.

[6] Benth, F. E., Karlsen, K. H., Reikvam K. (2003): Merton's portfolio optimization problem in a Black and Scholes market with non-Gaussian stochastic volatility of Ornstein-Uhlenbeck type. Math. Fin. 13(2), 215-244.

[7] Eberlein, E., and Keller, U. (1995): Hyperbolic Distributions in Finance, Bernoulli 1(3), 281-299.

[8] Emmer, S., Klüppelberg, C. (2004): Optimal Portfolios when Stock Prices Follow an Exponential Lévy Process. Fin. Stoch. 8, 17-44.

[9] Fleming, W. H., Hernández-Hernández, D. (2003): An Optimal Consumption Model with Stochastic Volatility. Fin. Stoch. 7, 245-262.

[10] Folland, G. (1999): Real Analysis, John Wiley \& Sons, Inc.

[11] Fouque, J.-P., Papanicolaou, G., and Sircar, K. R. (2000): Derivatives in Financial Markets with Stochastic Volatility. Cambridge: Cambridge University Press. 
[12] Goll, T., Kallsen, J. (2003): A Complete Explicit Solution to the LogOptimal Portfolio Problem. Ann. Appl. Prob., 13, 774-799.

[13] Ikeda, N., Watanabe, S. (1989): Stochastic Differential Equations and Diffusion Processes, 2nd ed. Kodansha: North-Holland.

[14] Korn, R. (1997): Optimal Portfolios, World Scientific Publishing Co. Pte. Ltd.

[15] Merton, R. (1969): Lifetime Portfolio Selection under Uncertainty: The Continuous Time Case. Rev. Econ. Stat. 51, 247-257.

[16] Merton, R. (1971): Optimum Consumption and Portfolio Rules in a Continuous Time Model. J. Econ. Theory 3, 373-413; Erratum (1973) 6, 213-214.

[17] Nicolato, E., Venardos, E. (2003): Option Pricing in Stochastic Volatility Models of the Ornstein-Uhlenbeck Type. Math. Fin. 13, 445-466.

[18] Pham, H., Quenez, M-C. (2001): Optimal Portfolio in Partially Observed Stochastic Volatility Models. Ann. Appl. Prob. 11, 210-238.

[19] Protter, P. (2003): Stochastic Integration and Differential Equations, 2nd ed. New York: Springer.

[20] Rydberg, T. H. (1997): The Normal Inverse Gaussian Lévy Process: Simulation and Approximation, Communications in Statistics: Stochastic Models 13(4), 887-910.

[21] Sato, K. (1999): Lévy Processes and Infinitely Divisible Distributions. Cambridge: Cambridge University Press.

[22] Øksendal, B. (1998): Stochastic Differential Equations, 5th ed. Berlin: Springer. 